Running head: MODELING INTRUSIVE MEMORIES IN PTSD

\title{
Memory, Interrupted: A Retrieval Model of Intrusive Memories, Recovery Trajectories, and Neurobiological Effects in Post-Traumatic Stress Disorder
}

\author{
Andrea Stocco ${ }^{1}$, Briana M. Smith ${ }^{2}$, \\ Emma PeConga ${ }^{1}$, Lori Zoellner ${ }^{1}$, \\ ${ }^{1}$ Department of Psychology, University of Washington, Seattle, WA, 98195 \\ ${ }^{2}$ Department of Bioengineering, University of Washington, Seattle, WA, 98195
}

Author Note:

Andrea Stocco, https://orcid.org/0000-0001-8919-3934

Emma Peconga, https://orcid.org/0000-0003-1831-3403

Lori Zoellner, https://orcid.org/0000-0002-7101-0703

The authors declare no conflict of interest

Correspondence should be addressed to: Andrea Stocco, Department of Psycology, Campus Box 351525, University of Washington, Seattle, WA, 98122. Email: stocco@uw.edu 


\begin{abstract}
Post-traumatic stress disorder (PTSD) is a psychiatric disorder often characterized by the unwanted re-experiencing of a traumatic event through nightmares, flashbacks, and/or intrusive memories. This paper presents a neurocomputational model using the ACT-R cognitive architecture that simulates intrusive memory retrieval following a potentially traumatic event (PTE) and derives predictions about an individual's recovery trajectory, behavioral symptoms, and neurological effects. Memory intrusions were captured in the ACT-R framework by weighting the prior probability of re-encoding a memory by an emotional intensity term $I$, which captures the degree to which an event was perceived as dangerous or traumatic. A series of simulations were run in which a model performed memory retrievals under naturalistic conditions for up to two months after experiencing a simulated PTE. It was found that $I$ had a significant effect on the probability of experiencing traumatic memory intrusions following a PTE, and that, under different conditions, the model experienced different probabilities of undergoing different recovery trajectories.
\end{abstract}

Keywords: Post-Traumatic Stress Disorder; Hippocampus; Amygdala; Declarative Memory; Long-Term Memory; ACT-R; Cognitive Architecture 


\section{Introduction}

Intrusive memories are emotionally-charged memories of traumatic events whose unwanted recollection disrupts normal function and causes additional stress. They are a transdiagnostic symptom across different mental health conditions, such as depression, schizophrenia, social anxiety disorder, and generalized anxiety disorder (Hagenaars et al., 2009). The time course of intrusive memories is important for understanding and predicting recovery trajectories, which remain highly variable and difficult to characterize in clinical practice and research.

Perhaps the most prototypical pathology characterized by intrusive memories is posttraumatic stress disorder (PTSD), a condition of severe distress triggered in response to a traumatic event. PTSD offers a model situation to study intrusive memories and understand the clinical significance, as its onset is typically tied to a specific event in time (the potentially traumatic event, or PTE) and intrusive re-experiencing of the event is an important part of its related symptomatology. Intrusive memories are distinguished from the normative re-experience of events because of their unwanted and uncontrolled nature, increased frequency, persistence over time, and the severity of distress that is associated with them (Bywaters et al., 2004).

The goal of this project is to use the tools of computational cognitive neuroscience to provide a mechanistic explanation of how intrusive memories arise and which factors affect the possible recovery trajectories. Specifically, this paper presents a computational model that describes a potential mechanism by which intrusive memories arise and makes predictions about their frequency over time, their possible recovery trajectories, and their biological and behavioral consequences. A central assumption that sets our approach apart from previous models (Radell et al., 2017) is that traumatic memories can be understood within the context of a general theory of 
episodic memory. This theory is grounded in a rational analysis of memory, is well specified computationally, and has a clear interpretation in terms of biology, thus providing a solid conceptual framework to understand and model traumatic memories.

This approach might seem paradoxical because, while most models of episodic memories stress its Bayesian rationality and the adaptiveness of remembering and forgetting processes (Anderson, 1990), intrusive memories are considered maladaptive, dysfunctional, and disruptive to a normal life. As the reminder of this paper will show, this paradox might be illusory: intrusive memories can be modeled following the same adaptive laws that regulate normal memories; however, the exceptional conditions under which traumatic memories are encoded lead the normally self-regulating mechanisms of forgetting to out-of-the-ordinary, selfperpetuating dynamics. Within this framework, the persistent memory intrusions observed in PTSD can be seen not as a maladaptive response, but rather as the runaway process of an otherwise adaptive memory system.

A traumatic memory tends to out-compete more contextually appropriate memories due to the fact it was encoded in a highly emotional state. With each retrieval of the traumatic memory, disproportionately more resources are allocated to it, leading to the further preservation and growth of these unwanted memory intrusions. Under different initial conditions, this positive feedback loop might not be initiated or, if it is initiated, might extinguish itself, stabilize, or worsen, leading to different recovery trajectories. Within this framework, it is proposed that the neurobiological changes following a traumatic event are the result of different computational demands posed to the biological memory circuitry. Specifically, potential changes in hippocampal volume associated with PTSD (O'Doherty et al., 2015) can be explained as the natural result of allocating neural resources to changing memory demands, and potential changes 
in the functional connectivity between the frontal cortex and the hippocampus (Shin et al., 2006) can be explained as an increased bottom-up, and reduced top-down, control over the contents of memories being retrieved.

The remainder of this paper is structured as follows. First, we will review the main findings on intrusive memories in PTSD, including some critical factors that are known to affect them. Second, we will introduce the model and provide an interpretation of its internal parameters in terms of cognitive constructs and neurobiology. Then, we will examine the model's performance across a series of parameter values, and examine the degree to which it correctly replicates the main findings in the literature in terms of behavioral and neurobiological findings. Finally, we will review the model, its limitations, and possible directions in which it could be refined.

\section{Intrusive Memories in PTSD}

Although intrusive memories appear as symptoms in multiple psychological disorders, this paper will focus on modeling their occurrence in PTSD. PTSD is usually understood as a persistent pathological response to an external event, the potentially traumatic event (PTE). The existence of such an external event provides a natural way to anchor the evolution of symptoms from a particular point in time, and makes PTSD a convenient starting point for any modeling effort that ties together memory processes and psychopathology.

In examining intrusive memories, two important outcomes need to be considered. One is the absolute outcome at a time point, which can be measured, for example, by the number of daily or weekly intrusive memories experienced by a patient. Their frequency, together with the characteristics of their context and the context in which they occur, are highly correlated with the severity of PTSD symptoms, explaining up to $43 \%$ of the variance in severity six months after 
the PTE (Michael et al., 2005).

The second outcome of interest is the patient's recovery trajectory, i.e whether the patient is on their way to improving or not. In a recent and influential review, Galatzer-Levy et al. (2018) identified four typical recovery trajectories following the PTE, which are illustrated in Figure 1. In this figure, the $x$-axis represents time, the vertical black line represents the onset of a PTE, and the $y$-axis represents the severity of intrusive re-experiencing on a qualitative scale. After a PTE, an individual might not show intrusive re-experiencing symptoms (resilient trajectory, green); show acute symptoms that spontaneously decline in severity over time (recovery trajectory, blue); exhibit worsening symptoms over time (delayed trajectory); or never recover after showing an initial acute response (chronic trajectory).

\section{Theoretical Recovery Trajectories}

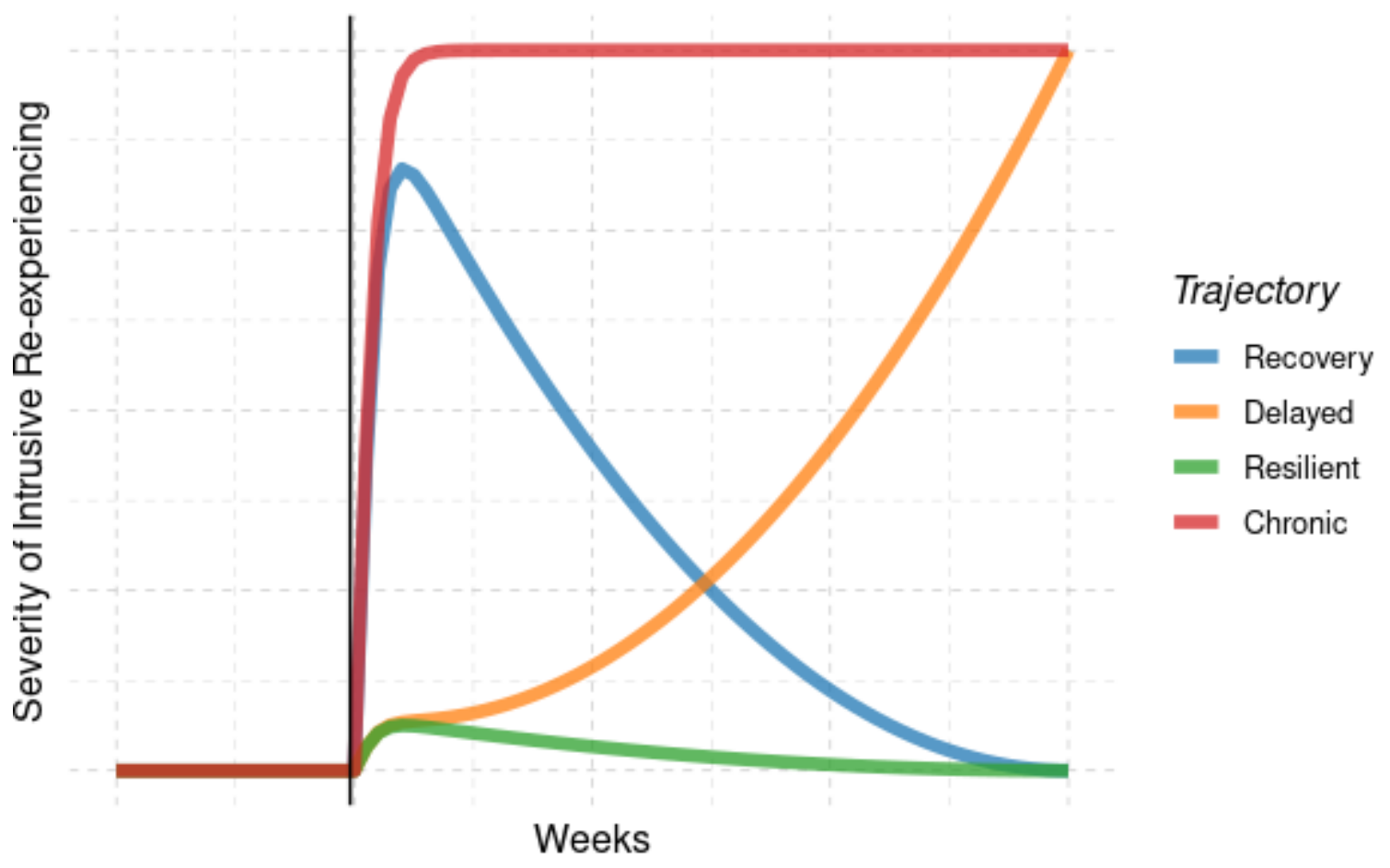

Figure 1: Idealized recovery trajectories after a PTE (black vertical line) 
Because these trajectories were derived from an overview of studies that differ greatly from each other, they are idealized in two important ways. First, all of the non-resilient trajectories appear to reach the same maximum level of symptoms on the $y$-axis, while this does not need to be the case. Second, an individual's trajectory might be categorized differently depending on the time scale at which it is observed. Different studies, for example, might examine clinical outcomes weeks, months, or years after the PTE; it is possible that an individual who is categorized as chronic two months after the PTE would be considered recovering one year afterwards, and that an individual exhibiting a resilient phenotype after one month could experience a delayed onset at a longer time scale.

These limitations notwithstanding, the existence for these different trajectories is generally strong, as variants of these trajectories can be found across a variety of studies and reviews, and at least three of them can be identified in animal model studies (evidence for the delayed onset trajectory is debated, and this trajectory does not typically appear in animal studies of fear conditioning). Importantly, the existence of different trajectories poses significant challenges for the early identification of those at high risk for chronic psychopathology—an important clinical goal not only for preventing long-term suffering but also for appropriately allocating limited resources. For this reason, it is important to characterize and understand the nature of the factors that might lead to these different patterns.

While some of the factors could be attributed to environmental effects, at least some of the differences in recovery curves must be due to the idiographic characteristics of the patients. Evidence in this sense comes from a review of studies on fear generalization and extinction in rodents (a common paradigm to study the neurobiology of PTSD), which concluded that at least three of the most common trajectories (chronic, spontaneous recovery, and resilience) also 
appear in animal models of PTSD (Galatzer-Levy et al., 2013).

\section{Factors Affecting the Frequency of Intrusive Memories Following a PTE}

In a recent review, Marks et al. (2018) provide an exhaustive examination of the possible factors that have been associated with the frequency and severity of intrusive memories. Many factors that had been suggested did not show more than modest effects in Mark's review. For example, trait dissociation did not consistently or robustly predict intrusive memories in most studies. Some of the factors that emerged as having significant effects, on the other hand, present significant difficulties for a modeling approach. For example, the presence of trait anxiety and depression were a small to moderate predictor of PTSD; yet, no consensus exists on how these pathologies should be modeled, as the field of computational psychiatry is still in its infancy (in fact, the present study is an attempt to advance this very field). Similarly, although gender is an important factor determining both the severity and the type of PTSD symptoms (Frans et al., 2005), it cannot be easily captured through model parameters. Finally, some factors that are robust predictors but are, in fact, correlated with other factors. For example, the tendency towards "data-driven" processing, defined as encoding sensory-perceptual details without the broader conceptual context of the event and typically assessed via self-report, does predict intrusive memories but likely occurs more in those with trait anxiety and increases as state anxiety increases.

Thus, the modeling efforts described herein focused on a handful of factors that (1) stand out as having a robust and well-documented effect, (2) can be naturally interpreted within our modeling framework, and (3) are representative of different categories. For simplicity, we will divide these factors into three categories: environmental factors, idiographic factors, and the tendency for negative re-appraisals, which is often tied to the presence of other conditions liek 
COMPUTATIONAL MODEL OF INTRUSIVE MEMORIES

anxiety and depression (Table 1).

\section{Environmental Factors}

We use the term "environmental factors" to indicate factors related to the characteristics of the PTE and their relationship to an individual. Two environmental factors were selected. The first is the emotional intensity of the traumatic event. The PTSD literature covers a vast variety of traumatic experiences, spanning the gamut from witnessing a crime to surviving torture. Although all of these events are traumatic, they vary in degree and severity, and these variations are reflected in their effects on the individuals affected by them. Traumatic events of greater perceived intensity tend to be associated with the worst outcomes (Frans et al., 2005), often in a dose dependent manner (Kolassa et al., 2010).

Traumatic events vary not only in their intensity, but also in the circumstances in which they might occur. These circumstances, and specifically the degree to which they are perceived to overlap with the daily routines, also profoundly affect the severity of PTSD symptoms. Sexual assault victims, for example, might have experieced trauma in their very own workplace or home, while the trauma experienced by combat veterans typically occurs in situations that have little overlap with the their civilian life. The similarity between the circumstances of the PTE and the context of everyday life plays an important role in triggering intrusive memories, since a more similar environment offers more contextual cues for retrieval (Besnard \& Sahay, 2016). 
Table 1 Factors affecting the frequency of intrusive memories in PTSD.

\begin{tabular}{|l|l|l|l|}
\hline \multicolumn{1}{|c|}{ Category } & \multicolumn{1}{|c|}{ Factor } & \multicolumn{1}{c|}{$\begin{array}{c}\text { Relationship to } \\
\text { Mental Health Outcome }\end{array}$} & $\begin{array}{l}\text { Model } \\
\text { Parameter }\end{array}$ \\
\hline Environmental & $\begin{array}{l}\text { Intensity of the } \\
\text { traumatic event }\end{array}$ & $\begin{array}{l}\uparrow \text { Greater intensity }= \\
\downarrow \text { worse outcome }\end{array}$ & $I$ \\
\cline { 2 - 4 } & $\begin{array}{l}\text { Similarity between } \\
\text { the PTE and daily } \\
\text { life events }\end{array}$ & $\begin{array}{l}\uparrow \text { Greater similarity }= \\
\downarrow \text { worse outcome }\end{array}$ & $C$ \\
\hline Idiographic & Cognitive control & $\begin{array}{l}\uparrow \text { Greater control }= \\
\uparrow \text { better outcomes }\end{array}$ & $W$ \\
\cline { 2 - 5 } & $\begin{array}{l}\text { Vividness of } \\
\text { recollection }\end{array}$ & $\begin{array}{l}\uparrow \text { Greater vividness }= \\
\downarrow \text { worse outcomes }\end{array}$ & $\gamma$ \\
\hline $\begin{array}{l}\text { Rumination or } \\
\text { Negative Appraisal }\end{array}$ & $\begin{array}{l}\text { Unproductive } \\
\text { Processing }\end{array}$ & $\begin{array}{l}\uparrow \text { Greater unproductive processing }= \\
\downarrow \text { worse outcome }\end{array}$ & $U$ \\
\hline
\end{tabular}

\section{Idiographic Factors}

It is generally understood that differences in outcomes are an interaction of

environmental and idiographic factors, that is, factors that capture the specific cognitive traits of a patient. This paper focuses on two of such factors. The first such factor is a patient's capacity to exert control over their own thoughts and habitual responses. This capacity is known as cognitive control or executive function and it is often referred to in the clinical literature (Bomyea \& Lang, 2015; Cohodes et al., 2020; Peverill et al., 2017)). Many PTSD studies have also focused on related psychological constructs, such as response inhibition (van Rooij et al., 2014; van Rooij \& Jovanovic, 2019), working memory (Saunders et al., 2015; Weber et al., 2005) and intelligence (Sopp et al., 2021); all of these concepts are highly overlapping (Miyake et al., 2000). However operationalized, the existing clinical research shows that individuals with better capacity for cognitive control experience less or less-severe PTSD symptoms, likely because of a better ability to manage or inhibit unwanted intrusive memories. 
COMPUTATIONAL MODEL OF INTRUSIVE MEMORIES

Another important factor is the vividness of the recollection experience, that is, the degree to which a retrieved memory carries the same emotional intensity as the original event. This factor also appears, under different studies, with different names. Some studies discuss it in terms of sensorimotor vividness, that is, greater detail and imagery in the re-experience of sensory details of the traumatic event (Berntsen et al., 2003; Morina et al., 2013). Other studies discuss it in terms of emotional vividness, typically in terms of the emotional intensity of the memory (Hackmann et al., 2004). In general, the vividness of the details of the memory is highly associated with its emotional intensity, so the two are considered the same herein.

\section{Unproductive Processing}

As noted in the previous sections, intrusive memories are trans-diagnostic and occur in multiple disorders. Conversely, the co-occurrence of other disorders represents a risk factor for PTSD, exacerbating the tendency to retrieve unwanted traumatic memories. This tendency to further retrieve and elaborate on negative thoughts and memories is categorized as either rumination or negative appraisal. Rumination is the tendency to perseverate on negative thoughts and feelings, whereas negative appraisal is the tendency to reflect negatively on previous events. Not surprisingly, for individuals whose response style is characterized by ruminative processing or negative appraisals of their traumatic memory, the clinical outcome is usually worse (Marks et al., 2018).

Although there are important differences between these two clinical concepts, they share the common nature of conscious and deliberate retrieval of memories, among which the traumatic ones are likely to figure prominently. Such a process has a clear connection to the mechanics of the model proposed here: by increasing the frequency with which a traumatic memory can be retrieved, both rumination and negative appraisal contribute to enhancing its 
availability and, therefore, the future probability of intrusive memories. For simplicity, the model proposed herein does not distinguish between rumination and negative appraisal, and we will refer to this common phenomenon simply as unproductive processing.

\section{Neurobiological Effects of PTSD}

In addition to psychological symptoms, trauma may also leave recognizable traces in the survivors' brain activity. A prevalent view in the literature organizes these changes in the context of the so-called Triple Network model, a framework that highlights changes in three interrelated networks of functionally and anatomically connected brain regions that are involved in controlling emotion and memory (Akiki et al., 2017). The first of these networks is the so-called salience network, which involves brain regions associated with quickly detecting the emotional relevance of external stimuli, including the insular cortex, the anterior cingulate cortex and, most notably, the amygdala. Hyperactivity in this network, and in particular in the amygdala, is associated with PTSD symptoms (Koenigs \& Grafman, 2009). For instance, compared to trauma-exposed controls, patients with PTSD symptoms show increased amygdala activity to negative stimuli (Shin et al., 2004, 2005, 2006).

The second network is the Default Mode Network (DMN), a large network of regions comprising the medial prefrontal regions, the medial parietal and retrosplenial cortex, and, crucially, the hippocampus. The hippocampus is necessary for long-term memory formation and storage of episodic and autobiographical memories (Moscovitch et al., 2005; Nadel et al., 2000; Nadel \& Moscovitch, 1997), although some researchers suggest that episodic memories might eventually be entirely transferred to neocortical sites (Alvarez \& Squire, 1994; McGaugh, 2000). In addition to the hippocampus, other regions in the DMN are involved in many aspects of episodic memory encoding and retrieval (Ranganath \& Ritchey, 2012). 
Intrusive memories are thought to occur because of the simultaneous engagement of the amygdala and hippocampus at the time of memory encoding, with amygdala re-activation later driving the spontaneous and recovery of the original traumatic event in the hippocampus (Marks et al., 2018). These spontaneous intrusions occur outside the scope of the usual mechanisms that control memory retrieval processes, which are associated with the third network: the centralexecutive network (CEN). This network spans dorsal prefrontal and superior parietal regions that are involved in higher-level control and coordination. Consistent with the presence of uncontrolled or involuntary memory intrusions, individuals suffering from PTSD exhibit reduced activity in regions belonging to this network, such as the medial prefrontal cortex (Shin et al., 2006).

Although the majority of studies has identified PTSD-specific changes in brain activity during task, the facets that are potentially more relevant to this paper are the long-term, stable changes that manifest themselves independently of the task, such as the possible changes in brain structure and in a spontaneous network activity.

At the anatomical level, PTSD is also generally characterized by a marked reduction in the volume of the hippocampus (Bremner et al., 1995, 1997; Logue et al., 2018; Nelson \& Tumpap, 2017; O’Doherty et al., 2015; Villarreal et al., 2002). It is important to note that this change is primarily structural, and, although often remarkably apparent, decreased hippocampus size is not consistently accompanied by a functional impairment in long-term memory performance (Anke et al., 2006). Nonetheless, reductions in hippocampal volume in the range of 5-26\% have been observed across multiple studies (O’Doherty et al., 2015). Only a few studies have been able to examine hippocampal volume prior to trauma exposure and PTSD (Gilbertson et al., 2002; Pitman et al., 2006). When looking cross-sectionally, the majority of studies suggest 
that changes in hippocampal volume may be a neurological manifestation of PTSD. The model reported herein provides an explanation for this finding, relating the change in structure to the differential neural resources required to accommodate intrusive memories.

A second important change occurs in the resting-state functional connectivity between different regions in the default mode network. Resting-state functional connectivity is measured as the correlation in low frequency $(0.1-0.01 \mathrm{~Hz})$ oscillations in spontaneous activity in the brain, which are recorded while participants are awake, but not actively doing any particular task, in an fMRI scanner. Thus measured, functional connectivity is stable and replicable, and assumed to index the spontaneous architecture of brain networks. Compared to healthy or traumatized, asymptomatic controls, individuals with PTSD show decreased functional connectivity between the medial prefrontal cortex and the hippocampus. This change is believed to reflect a reduced capacity of top-down, goal-oriented control on the contents of memories that are being retrieved (Koenigs \& Grafman, 2009; Shin et al., 2006).

\section{Intrusive Memories and Existing Models of PTSD}

As noted above, the biological mechanisms of PTSD are typically understood through the lens of the fear conditioning and extinction literature. In computational models, conditioning and extinction are captured through Reinforcement Learning (Sutton \& Barto, 1998), a formal theory of how agents learn to select different actions based on environmental feedback (Myers et al., 2013; Radell et al., 2015, 2016; Radell et al., 2017; Sheynin et al., 2015). For example, Myers et al. (2013) developed an RL model with separate mechanisms for rewards and losses, and showed that performance of PTSD participants in a probabilistic decision-making task, could be modeled as a consequence of abnormally large reward values ascribed to neutral trials. A related model (Radell et al., 2016) showed similar results in groups of participants with the personality trait of 
behavioral inhibition, typically marked by higher anxiety and avoidance behavior. Individuals with high behavioral inhibition showed higher reward values assigned to neutral faces in a trust task than individuals with lower inhibition. Both findings can be interpreted as related to negative expectations connected to avoidance and impaired performance in learning from rewards, both of which are potentially implicated in PTSD.

As noted by Radell et al. (2017), these models are rarely designed to capture individual differences. They are also geared towards capturing laboratory-based paradigms rather than naturalistic circumstances, and task-based performance measures rather than clinical outcomes such as recovery trajectories or estimates of clinical symptoms. To the best of our knowledge, none of these existing models focuses specifically on intrusive memories nor captures long-term memory dynamics and clinical symptoms. These dynamics, instead, were explicitly included in an influential framework proposed by Rubin et al. (Rubin et al., 2008). Rubin's theoretical model shares the same viewpoint as the present paper but lacks a computational implementation and, therefore, yields only qualitative predictions. Thus, the model proposed herein offers a new perspective to the study of intrusive memories in PTSD from a computational neuroscience viewpoint.

\section{Summary and Goals}

The brief review of the literature outlined above clearly points to the reasons why a new model is needed. Effective mental health preventative care and treatment measures depend on the ability to successfully predict recovery trajectories, but the ability to predict recovery trajectories is hindered by the existence of multiple factors that might affect the outcome. To further complicate the problem, these factors are often difficult to measure objectively and their relationship with the outcome might be indirect and non-linear. 
Computational models are a theory-driven approach to capture the effects of the various factors and constrain their inter-dependencies into a principled set of relationships and assumptions (White et al., 2014, 2016). In addition, if computational models are rooted in cognitive and neurobiological frameworks, their internal parameters naturally reflect established and measurable constructs, facilitating their interpretation of their effects on patient mental health (Huys et al., 2016; Redish, 2004). Finally, a computational model might provide a principled way to quickly test and revise assumptions in developing future, better-targeted PTSD prevention interventions and treatments.

The next section will provide an overview of the proposed model, explaining how the model relates to an existing model of episodic memory; how these models can be extended to account for the specific characteristics of PTSD; and how the parameters governing the model relate to concepts rooted in both the neurocognitive and clinical literature.

\section{A Computational Framework for Traumatic Memories}

The model presented herein is based on ACT-R's theory of declarative memory (Anderson, 2007). This choice was motivated by three reasons. First, ACT-R is currently the most commonly adopted cognitive architecture in psychology and cognitive neuroscience (Kotseruba \& Tsotsos, 2018). Second, ACT-R has a long and established history of application to brain sciences, making the process of drawing new inferences at the neural level easier and less tentative. Finally, ACT-R is based on a Bayesian framework, which provides an elegant foundation of declarative memory retrieval processes and can be extended to incorporate the proposed theory of memory retrieval according to their emotional intensity.

Despite previous attempts (Dancy, 2013; Fum \& Stocco, 2004; Juvina et al., 2018), ACT$\mathrm{R}$ currently lacks a formal theory of emotion. Thus, it is not possible to implement the effects 
that traumatic experiences have on memory without extending the underlying theory. To provide a rational justification for such an extension, we will follow the Bayesian interpretation of memory that was the original inspiration for ACT-R's memory system (Anderson \& Schooler, 1991).

In the rational analysis terms pioneered by Anderson (1990), a memory's retention function (that is, its availability across contexts and times) reflects its probability of being needed at a given moment. Thus, if we indicate the specific memory as $m$ and the context as $Q$ (composed of different elemental cues $q_{1}, q_{2}, \ldots q_{n}$ ), a memory retention function reflects the posterior odds $P(m \mid Q) / P(\neg m \mid Q)$, which can be expressed as the product of the prior odds $P(m) /$ $P(\neg m)$ and the likelihood $P(Q \mid m) / P(\neg Q \mid m)$. Assuming, for simplicity, that each cue $q$ is independent from each other, the retention function can be expressed in terms of log odds as:

$$
\begin{aligned}
\log \left(\frac{P(m \mid Q)}{P(\neg m \mid Q)}\right) & =\log \left(\frac{P(m)}{P(\neg m)} \times \frac{P(Q \mid m)}{P(Q \mid \neg m)}\right) \\
& =\log \left(\frac{P(m)}{P(\neg m)}\right)+\log \left(\frac{P(Q \mid m)}{P(Q \mid \neg m)}\right) \\
& =\log \left(\frac{P(m)}{P(\neg m)}\right)+\log \left(\prod_{q} \frac{P(q \mid m)}{P(q \mid \neg m)}\right) \\
& =\log \left(\frac{P(m)}{P(\neg m)}\right)+\sum_{q} \log \left(\frac{P(q \mid m)}{P(q \mid \neg m)}\right) \\
& =\log \left(\frac{P(m)}{P(\neg m)}\right)+\sum_{q} \log \left(\frac{P(q \mid m)}{P(q)}\right)
\end{aligned}
$$

The last step in Eq. 1 is an approximation derived from the consideration that, for large amounts of memories, $P(q \mid \neg m) \approx P(q)$. The terms in Eq. 1 have a straightforward explanation in terms of the cognitive psychology of memory (Anderson, 1990; 2007; Anderson et al., 2004). 
Specifically, the log posterior odds on the left-hand side of Eq. 1 correspond to a memory's activation, an intuitive construct that describes a memory's moment-to-moment availability. Similarly, the two quantities on the right-hand side also correspond to cognitive constructs, with the log priors corresponding to the base-level activation of $m$ or $B(m)$ (capturing the effects of the previous history of usage of $m$ ) and the log-likelihood corresponding to the contextual or spreading activation of $m$, or $S(m)$ (capturing the additive effects that each environmental cue has on the memory's activation). Thus:

$$
\begin{aligned}
A(m) & =\log \left(\frac{P(m \mid Q)}{P(\neg m \mid Q)}\right) \\
B(m) & =\log \left(\frac{P(m)}{P(\neg m)}\right) \\
S(m) & =\sum_{q} \log \left(\frac{P(q \mid m)}{P(q)}\right)
\end{aligned}
$$

and, therefore, $A(m)=B(m)+S(m)$.

To implement these equations algorithmically (Anderson, 2007), the quantities $B(m)$ and $S(m)$ are approximated in ways that predict future use based on the previous history or the learned associations between the memory and the contextual cues, respectively.

In general, the probability of retrieving a memory declines over time according to a power function (Anderson \& Schooler, 1991). Assuming that every single use of a memory $m$ corresponds to the creation of a new trace (Nadel et al., 2000) for $m$, and that the probability of each trace being retrieved declines over time according to the same power function with a decay rate $d$, then the odds of retrieving $m$ are the odds of retrieving any of the traces associated with $m$, or the sum of the probabilities of each trace being retrieved. Because activation is expressed 
in the form of log odds, the value of $B(m)$ at time $t$ is the log of the sum of decaying traces associated with $m$ 's usage:

$$
B(m)=\log \left(\sum_{j}\left(t-t_{j}\right)-d\right)
$$

in which $t_{j}$ represents the time at which trace $j$ has been encoded, and $t-t_{j}$ is the age of the trace. Fig. 2 provides a visual illustration of this mechanism, assuming the same memory has four traces associated with it, generated at times $t_{1}=0$ (memory creation), $t_{2}=20 \mathrm{~s}, t_{3}=55$, and $t_{4}$ $=65 \mathrm{~s}$.

The model is noteworthy for its reliability, having been used to successfully model effects of recency, frequency, and spacing in declarative learning (Anderson et al., 1998; Anderson, 1974; Pavlik \& Anderson, 2005) and having been used to successfully derive optimal schedules for learning practice (Pavlik \& Anderson, 2008). The decay parameter $d$ in Eq. 3 has been also used for deriving idiographic parameters (Sense et al., 2016), with $d$ being a stable trait within the same individual across sessions and materials, and to assess individual differences in real-life outcomes, such a student's success at answering test questions after studying (Sense et al., 2018). Notably, these studies have shown that the same model and the same parameter values can be used to capture effects at different time scales, from minutes in laboratory settings (Pavlik \& Anderson, 2005) to weeks and months in naturalistic tasks (Sense et al., 2018). 


\section{Individual Traces}

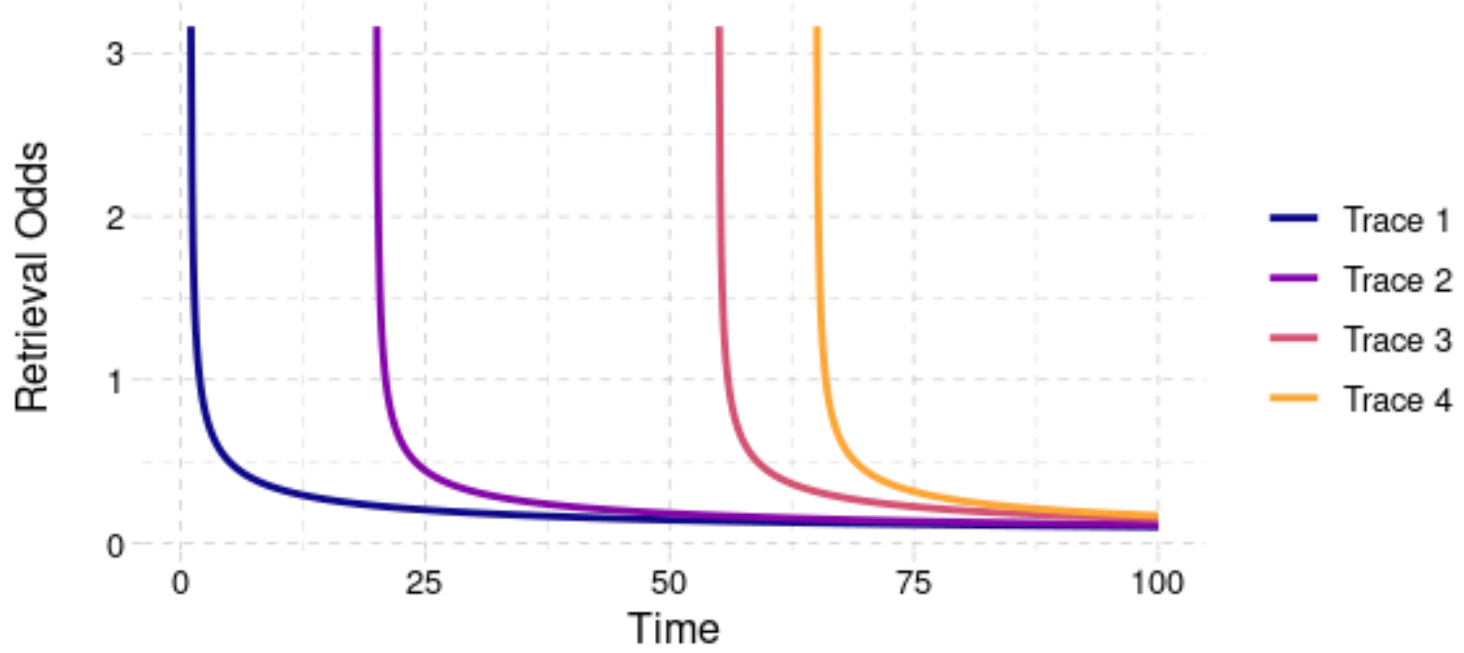

\section{Memory}

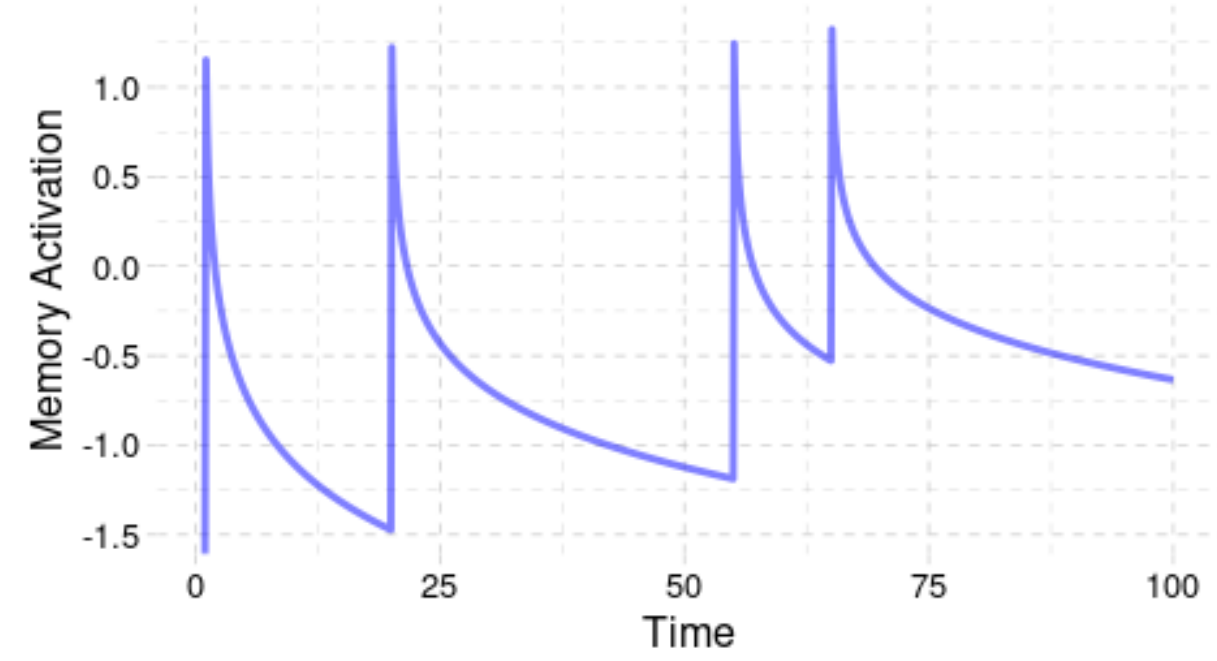

Figure 2. Time course of base-level activation of a memory $\mathrm{m}$ which has four traces associated with it, encoded at times $\mathrm{t}=0,20,55$, and $65 \mathrm{~s}$ (top). Each trace, shown in a different color, decays at the same rate $(\mathrm{d}=0.5)$. The odds of retrieving $\mathrm{m}$ are the sum of the odds of retrieving any of its traces (bottom). 
The base-level activation component provides a first clue as to the reason why intrusive memories exist. As shown in Figure 2, the more a memory has been retrieved, the more of its traces are created and the more likely it is to be retrieved in the future. Intrusive memories feed off this frequency effect because more unwanted retrievals in the past lead to more unwanted retrievals in the future. Under normal circumstances, this cycle is broken by the natural decay of each trace (the recency effect). As it will be shown, the special circumstances under which traumatic memories are created, however, makes them more likely to become persistently intrusive. The effect of previous retrievals is also related to one of the factors affecting the frequency of intrusive memories, namely, the effect of negative appraisal. This is because a tendency to revisit negative events would likely result in more retrievals of a traumatic memory, ultimately increasing its odds of intruding in the future.

Spreading activation $S(m)$, on the other hand, can be best understood in reference to a classic representation format for memories, namely, semantic networks (Anderson, 1983; Collins \& Loftus, 1975; Roediger et al., 2001; Roelofs, 1992). In semantic networks, each memory represents a node, and associated memories. are connected by directional links. Spreading activation $S(m)$ is implemented as the amount of activation that flows from the memory nodes that are part of the context $Q=\left\{q_{1}, q_{2} \ldots q_{N}\right\}$. Thus, each element $q_{j}$ represents a specific active node in the current context. The strength of the link between $q$ and $m$ reflects the statistics of cooccurrence between the two events, so that greater co-occurrence of $q$ when $m$ is present (i.e., $P(q \mid m))$ correspond to stronger links. If there is a direct link from $q$ to memory $m$, then $i$ receives an activation boost that is proportional to the product between the strength of the link connecting $q$ to $m$ (indicated as $S_{q \rightarrow m}$ ) and the attentional weight given that cue. The attentional weight is usually simplified as a single scalar quantity, $W$. The total amount of spreading activation $S_{i}$ that $i$ 
receives is the sum of all of the partial effects of each element $q$ :

$$
S(m)=\sum_{q=1}^{N} W S_{q \rightarrow m}
$$

The idea that spreading activation is related to attention has a long tradition in cognitive psychology (Anderson, 1983; Collins \& Loftus, 1975; Roelofs, 1992). Although the most common interpretation of the weight parameter $W$ is in terms of top-down attentional control in retrieval (Anderson, 1983), this parameter can also be interpreted as capturing executive functions and working memory (Burgess et al., 2011; Kane \& Engle, 2002, 2003), that is, an individual capacity to maintain, process, and update short-term information (Baddeley, 2010; Baddeley \& Logie, 1999). For example, Lovett and colleagues (Daily et al., 2001; Lovett et al., 2000) showed that individual variations in $W$ values capture idiographic differences in working memory performance and that $W$, when estimated independently through a working memory task, predicts performance on other tasks that demand cognitive control. Similarly, Rice and Stocco (2021) showed that individual variations in performances in the $N$-back task (a common measure of working memory) can be explained by corresponding differences in the $W$ parameter of the corresponding model.

Thus described, spreading activation is related to two important factors that affect PTSD. The first is the contextual similarity. In this framework, the degree of similarity between the context in which the PTE occurred and the context of daily activities is captured by the amount of individual cues $q$ that are shared between the two; The greater the number of shared cues, the greater is the spreading activation and thus the probability of involuntarily retrieving a memory related to the PTE. 
The second factor is executive function. In this framework, greater executive function can be translated into greater values of the parameter $W$. The greater is $W$, the greater is the amount of top-down control exerted over the retrieval process and, correspondingly, the smaller is the probability of involuntarily retrieving a traumatic memory in a different context.

Although spreading activation provides a way to capture these factors, two more factors (emotional intensity and recollection vividness) still need to be accounted. To do so, the model will need to be expanded to account for the effects of emotion on memory.

\section{Extending the Rational Analysis Framework}

As noted by Anderson (2007), classic approaches to memory, as summarized above, assume that all memories are perfectly equal in terms of their importance. This is highly unlikely to be the case; instead, multiple studies show that the amount of availability of a memory also reflects its intrinsic importance, even when recency, frequency, and contextual factors are accounted for. For example, stimuli associated with higher monetary rewards are remembered better than those associated with lower monetary rewards (Murty et al., 2016, 2017; Patil et al., 2017) and emotionally arousing stimuli memorized under stressful conditions tend to be remembered better than stimuli memorized under non-stressful conditions (Cahill et al., 2003).

A simple and coherent way to overcome this limitation of the classic rational analysis framework is by assuming that the availability of a memory reflects not only the mere probability, but the product of a memory's probability and its importance, which would be captured by the emotional effect at the time of encoding. This product is analogous to the concept of expected utility in decision-making (Schoemaker, 1982), which is defined as the product of an outcome's probability by its utility. In this case, a memory represents an outcome, its probability is the memory's posterior probability of being re-encoded, and its "utility" is its 
importance (Smith et al., 2021). Following on ideas of rational analysis, a memory's "importance" is supposed to reflect its utility for the survival of an organism; in this sense, although not important memories are necessarily tied to dangerous events and negative stimuli, memories for dangerous and fearful situations are intrinsically more "important" because they are needed not to incur in the same danger again (Ohman \& Mineka, 2001).

Thus defined, the importance of a memory can be captured by an term, $0<I(m)<\infty$, which represents the degree to which it is perceived as potentially dangerous or traumatic. Specifically, we will indicate a memory's importance as $I(m) / I(\neg m)$, or the ratio between $m$ 's importance and the mean importance of all other memories that are not $\mathrm{m}$, i.e. $I(\neg m)=\sum_{i \neq m}^{M}$ $I(i) / N$. As the number of memories grows, $I(\neg m)$ approximates the mean importance of all memories, $\bar{I}$. We can rewrite Eq. 2 expressing a memory's activation $A_{m}$ as its posterior probability multiplied by its importance $I(m) / I(\neg m)$ :

$$
\begin{aligned}
A(m) & =\log \left(\frac{P(m \mid Q)}{P(\neg m \mid Q)} \times \frac{I(m)}{I(\neg m)}\right) \\
& =\log \left(\frac{P(m)}{P(\neg m)} \times \frac{P(Q \mid m)}{P(Q \mid \neg m)} \times \frac{I(m)}{\bar{I}}\right) \\
& =\log \left(\frac{P(m)}{P(\neg m)}\right)+\log \left(\frac{P(Q \mid m)}{P(Q \mid \neg m)}\right)+\log \left(\frac{I(m)}{\bar{I}}\right) \\
& =\log \left(\frac{P(m)}{P(\neg m)}\right)+\log \left(\prod_{q} \frac{P(Q \mid m)}{P(Q \mid \neg m)}\right)+\log \left(\frac{I(m)}{\bar{I}}\right) \\
& =\log \left(\frac{P(m)}{P(\neg m)}\right)+\sum_{q} \log \left(\frac{P(q \mid m)}{P(q \mid \neg m)}\right)+\log I(m)-\log \bar{I} \\
& =B(m)+S(m)+\log I(m)-\log \bar{I}
\end{aligned}
$$


In realistic situations in which a large number of memories exist, the term log $\bar{I}$ can be approximated as a constant and can therefore be ignored from now on.

\section{Interpreting $I(m)$ in Terms of Emotions}

Crucially, the new term $I(m)$ can be interpreted in reference to emotional processing. A common theme across affective neurosciences is that emotions, at least in their most basic forms, are needed for survival (Ledoux, 1998; LeDoux, 2012; Panksepp, 2004). Therefore, events that are associated with different emotions, or to the same emotion but to a different degree, should also differ in survival value. For example, for a prey animal, the memory of a place where a previous encounter with a predator has occurred is more helpful for survival than the memory of the same place if no predator attack had occurred. Thus, the emotional state associated with an episodic memory should translate to its importance.

Although authors disagree on how to categorize emotions, most authors agree that emotions can be placed at least across two dimensions, valence and arousal---the so-called circumplex model of emotions (Posner et al., 2005). The term $I(m)$ can be thought of as the norm of the vector in this bidimensional space, capturing an emotion's survival value into a single metric. 


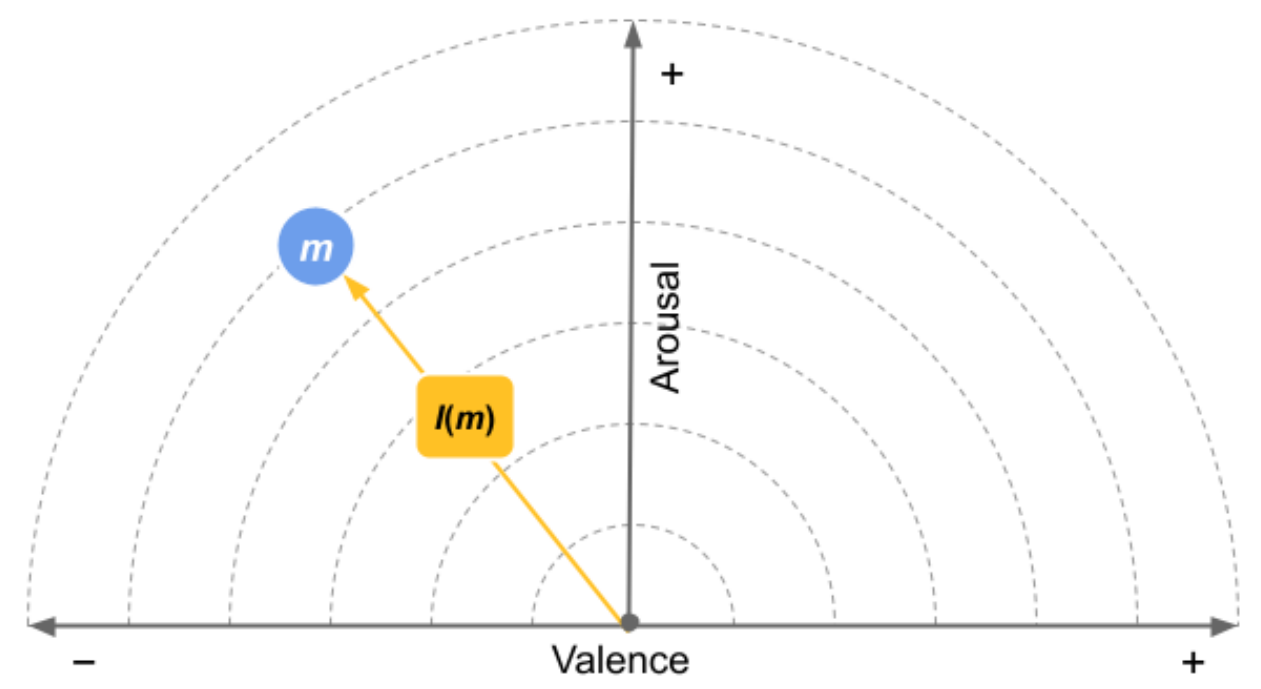

Figure 3. An interpretation of the $I(m)$ term in Eq. 3 within the circumplex model of emotions: $I(m)$ represents the combined effects of arousal and valence associated with memory $\mathrm{m}$.

\section{Emotional Re-Appraisal of Events}

The simple form of Eq. 3 assumes that the emotional intensity $I(m)$ is identical for every single trace of $m$. This is, of course, not true. As noted above and shown in Figure 4, the retrieval of a traumatic memory results in the creation of a new trace, and each new trace would have its own intensity $I$ that reflects the specific contribution of the amygdala at that moment in time. Thus, a realistic model needs to capture how I would change after each subsequent re-encoding of the traumatic memory.

The new I associated with the retrieval of a traumatic memory would likely be a combination of the $I$ value that has been retrieved and the value that is being experienced as part of the environment. For instance, Iuvina et al. (2018) have suggested that such adjustments follow the temporal-difference learning algorithm commonly used in Reinforcement Learning. In this case, we will assume that the emotional intensity of each trace declines with each 
retrieval, asymptotically reaching the mean intensity value across all memories, $\bar{I}$. A new trace, thus, would have a new intensity value $\gamma[I(m)-\bar{I}]$. The parameter $0<\gamma<1$ represents the vividness of a memory's recollection, and $[I(m)-\bar{I}$ represents the additional intensity of $\mathrm{m}$ above the average $\bar{I}$. As the quantity $[I(m)-\bar{I}$ gets closer and closer to zero, the intensity of a memory approximates the average of all memories.

If $n$ traces of $m$ are available, the expected value $I(m)$ at the $n$-th retrieval can be estimated as the mean intensity of all traces:

$$
I(m)=\sum_{i=1}^{n} \gamma^{i}[I(m)-\bar{I}] / n
$$

This quantity can be approximated by the power sum expression:

$$
\left.I(m)=[I(m)-\bar{l}]\left[\gamma\left(1-\gamma^{n}\right)\right] /(1-\gamma)\right] / n
$$

Thus defined, the parameter $\gamma$ accounts for the last remaining factor that affects intrusive memories, that is, memory vividness. For low values of $\gamma$, the emotional intensity associated with the retrieval of a traumatic memory quickly declines, causing a less vivid re-experience of the event. On the other end, as $\gamma$ approaches 1 , the emotional intensity of a memory declines less and less, and each retrieval is accompanied by a more vivid re-experience of the original event.

\section{Neurobiological Interpretation of the Model}

The model outlined above can also be interpreted in terms of brain circuitry. In this interpretation, the framework is broadly consistent with the Multiple Trace Theory of memory consolidation (Moscovitch et al., 2005; Nadel et al., 2000), a neural model that posits that every 
experience forms an episodic trace that is encoded in the hippocampus and that semantic memories are the result of multiple, partially overlapping traces creating stable and accessible patterns of neural activity.

\section{Base-level Activation and Hippocampus}

In this interpretation, the individual traces that make up every single memory in Eq. 3 correspond to the episodic traces of the MTT, and are encoded in the hippocampus, a subcortical region in the medial temporal lobe that is responsible for the encoding of memories (Figure 4, blue). The hippocampus receives topologically organized projections from all over the cortex, and (shown in blue in Figure 4), in turn, sends projections back. The hippocampus is well positioned to perform this encoding because its neurons form a densely interconnected network. It has been suggested multiple times that this particular pattern of connectivity can be interpreted as follows: the hippocampus works as an autoassociator or an autoencoder, a form of contentaddressable memory that stores patterns of cortical activity that can then be re-created based on partial inputs. This combination of features makes the hippocampus capable of fast learning and high-storing capacity, making it is complementary to to the slow learning that takes place in the cortex (McClelland et al., 1995). The decaying term $B_{i}$ reflects the progressive weakening of the hippocampal traces, due to either accumulated interference, biological decay (Alvarez \& Squire, 1994; Hardt et al., 2013), or a combination of both. 


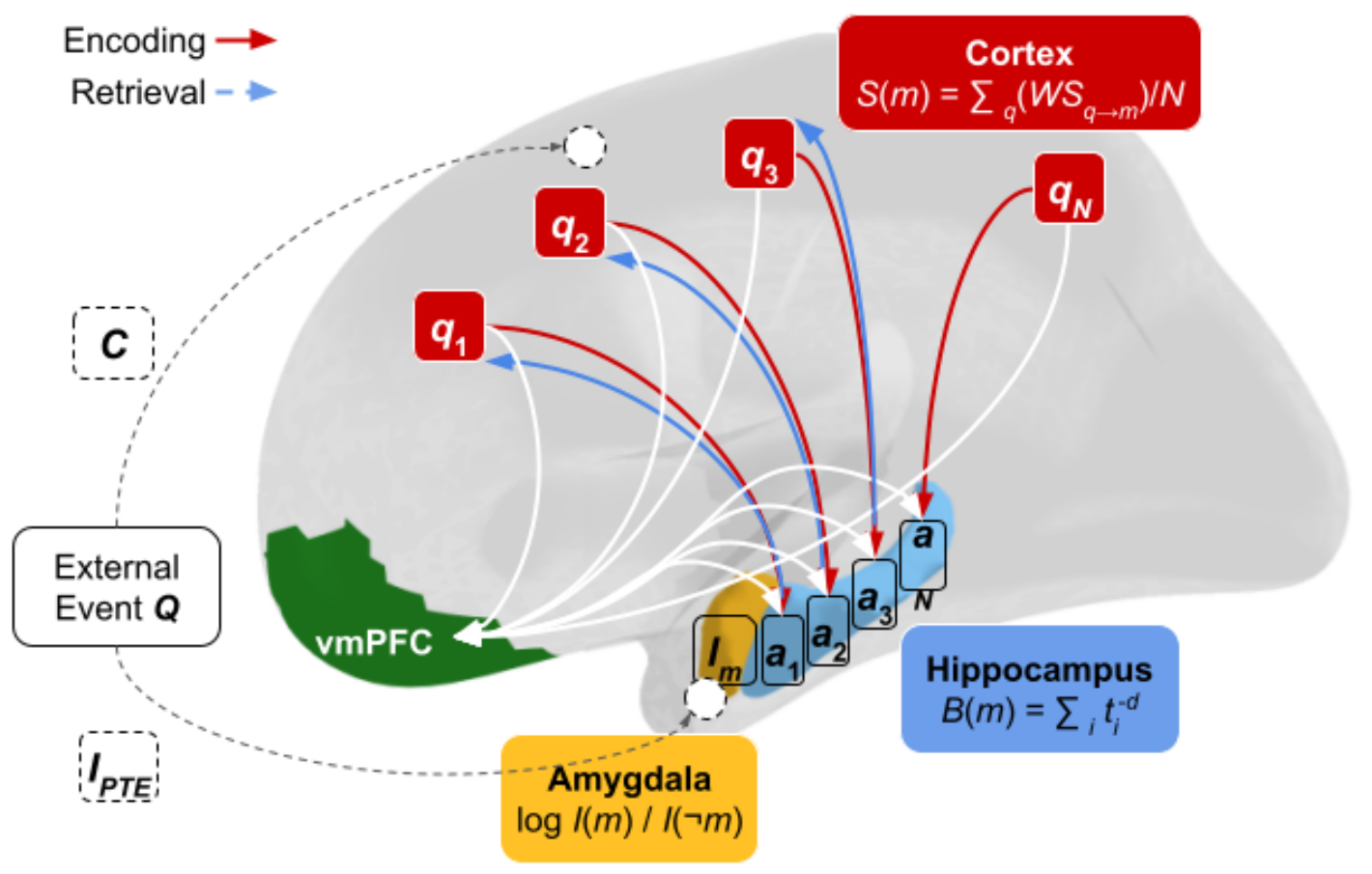

Figure 4: Neurobiological interpretation of the model components, their functional contribution, and their main parameters. In white, an external event, $Q$, is processed and represents the current context under which a memory will be retrieved. This context is represented by a number of individual sensory cues $q_{1}, q_{2} \ldots q_{N}$ that are individually represented in different cortical areas. The overlap between the current context and the context under which the traumatic memory was experiences is captured by the parameter $C$. The retrieval of an appropriate memory is controlled by the ventromedial prefrontal cortex (green) and the affected by the contributions of three components and their respective neural circuits: (1) the base-level activation, due to the intrinsic processes occurring in the hippocampus (in blue); (2) the spreading activation, due to the signals traveling over the pathways connecting cortical areas and the hippocampus (in red); and (3) an additional emotional boost that conveys the importance of a memory and is due to the activity of the amygdala (in yellow), 
An important biological effect of PTSD is the potetial presence of a reduction in hippocampus size. This small to moderate-size effect has been observed numerous times (Bremner et al., 1995, 1997; Villarreal et al., 2002). The model can be used to estimate the hippocampus size. As a proxy for hippocampus size, we calculated the entropy. This method was described in detail in Smith et al. (2021). In their formulation, the size of the hippocampus is related to the number of memories and their relative activation level. This relationship assumes that the hippocampus allocates neural resources (synapses and neurons) efficiently. The degree of efficiency is a function of the information entropy (Shannon, 1948) across all $M$ memories in long-term memory.

To calculate the entropy, it is necessary to transform each memory's activation into a corresponding probability value. Because, according to rational analysis, a memory's activation corresponds to its log odds of retrieval, the corresponding probability can be calculated as the ratio of a memory's odds against the odds of all memories:

$$
P(m)=\frac{e^{B(m)+I(m)}}{\sum_{j=1}^{M} e^{B(j)+I(j)}}
$$

The long-term memory entropy $H$ can then be calculated as:

$$
H=-M \times \sum_{j=1}^{M} P(i) \times \log P(i)
$$

\section{Spreading Activation and Cortical Projection}

Biologically, the retrieval of memories proceeds in a direction that is symmetric and 
opposite to the encoding process, with partial inputs from the cortex triggering the re-creation of a pattern of activity in the cortex (Danker \& Anderson, 2010; Moscovitch et al., 2005; Nadel et al., 2000). This process and its components are shown in red in Figure 4. The spreading activation term $S$ captures the role of cortical projection as retrieval cues, with different regions encoding different features and activating different possible traces in the hippocampus. The parameter $W$ determines the strength of top-down control of retrievals. The effect of $W$ can be put into correspondence to the activity of the inferior and medial areas of prefrontal cortex in the control of access to retrieval (Badre \& Wagner, 2007; Danker et al., 2008; Thompson-Schill et al., 1997). These processes are colored in white in Figure 4.

Biologically, the degree of this control is reflected in the degree of synchronization between medial prefrontal regions and the hippocampus (Shin et al., 2004, 2006). A computational proxy for this measure is the similarity between context $Q$ and the retrieved memory $m$, with less control associated with lesser similarity.

\section{Amygdala and Emotional Intensity}

The final piece in this circuit is the amygdala, a small nucleus located in front of the hippocampus. As noted above, the amygdala is part of the salience network, a network of regions known to play a role in recognizing possible threatening stimuli and which is hyper-active at rest in PTSD. The amygdala, in particular, plays a fundamental role in processing fear (Ledoux, 1998), and is bidirectionally connected to the hippocampus. Like the hippocampus, it receives widespread cortical projections from the cortex, which facilitates the immediate recognition of threatening circumstances. In our model, the functional role of amygdala corresponds to the term $I(m)$, which serves both as an emotional marker for a specific memory $m$ and as a cue that boosts its probability of being retrieved. 


\section{Summary and Predictions}

In the previous sections, we have outlined a model of how emotional memories can be integrated in an existing computational framework for long-term memory; how an event's emotional intensity boosts the probability of its memory to be retrieved; and how traumatic memories might become intrusive because of this additional boost. Additionally, we have also outlined how this model is compatible with the architecture of the brain circuits supporting emotion, memory, and retrieval, and how this framework could be used to explain hippocampal volume and functional connectivity between prefrontal cortex and the hippocampus.

The rest of this paper will describe an extensive analysis of this model and of how various factors, manipulated as model parameters, affect the model's outcomes in terms of simulated behavior and simulated brain dynamics.

\section{Material \& Methods}

\section{Model Implementation}

An algorithmic version of the model was implemented in the ACT-R cognitive architecture. ACT-R is a natural choice, since its declarative memory system reflects the Bayesian analysis that is the basis of Eq. 2 and only minor modifications were needed to implement the new activation equation Eq. 3. Furthermore, because ACT-R is currently the most popular cognitive architecture in psychology (Kotseruba \& Tsotsos, 2020), our code could be easily integrated in other models of memory. Finally, ACT-R is noteworthy because many of its modules and parameters have been put into correspondence with underlying neural circuits (Anderson et al., 2004; 2008; Stocco \& Anderson, 2008), thus providing an additional level of plausibility to our model. The entire code for the model, as well as the complete results of the simulations and all of the analysis, is available at https://osf.io/qpfr4/. 
COMPUTATIONAL MODEL OF INTRUSIVE MEMORIES

\section{Model Behavior}

The model simulates behavior over a period of time that spans several months, thus stretching the boundaries of a typical ACT-R model's timescale. The model follows a simple perceive-retrieve-respond loop, in which the model faces external events by retrieving a memory of a previously encountered situation and using this memory to generate a response. The outline of such a procedure is illustrated in Figure 4. Throughout a series of simulated days, the model is presented with a stream of new events. Each event, in turn, becomes the current context $Q$ and its elements become the current contextual cues $q_{1}, q_{2} \ldots q_{\mathrm{N}}$. During the perception phase, the model attends the event, making the cues become the source of spreading activation. The values of $B(m), S(m)$, and $I(m)$ are computed for every element in long-term memory (LTM). $B(m)$ is easily computed from the past history of encodings and retrievals of every memory. $S(\mathrm{~m})$ is computed by calculating the match between each contextual cue $q$ and the corresponding feature of every other event in LTM. Finally, $I(m)$ is calculated for every event in memory by looking up its corresponding entry in a corresponding table. During the retrieval phase, the memory with the highest activation value is then selected through a noisy soft-max procedure, and is placed in a special retrieval buffer, marking the end of the retrieval phase. Eventually, during the response phase, a response is performed based on the contents of the retrieval buffer, and the internal representation of the context $Q$ is encoded as a new memory in LTM. 


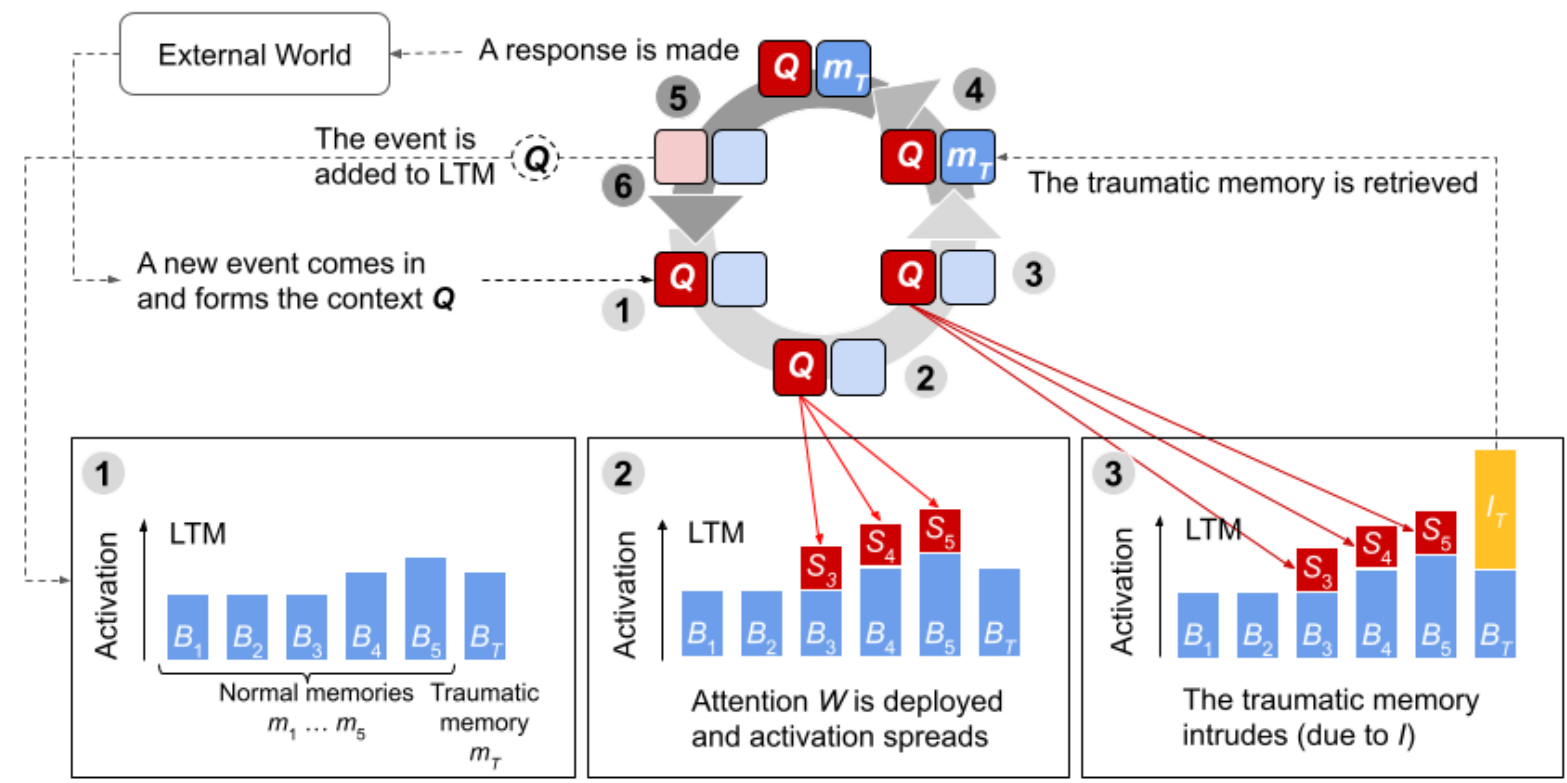

Figure 5: Overview of the model's behavior during a traumatic memory intrusion. The model implements a simple Perceive/Retrieve/Respond loop, and uses separate buffers to store contextual information $(Q$, red) and the retrieved memory (m, blue). (1) Initially, the availability of memories only reflects their base-level activations B. After an external event is presented, it is encoded to form the current response context $Q$. (2) Spreading activation from $Q$ changes the landscape of the model's long-term memory (LTM), varying the activation level of different memories to reflect their current relevance. (3) The additional emotional intensity $I_{T}$ of a traumatic memory $m_{T}$ provides and additional activation boost. (4) Because of its additional activation, the traumatic memory $m_{T}$ intrudes in the retrieval process even if it is the contextually inappropriate response to the current event. (5) Eventually, the model's memory is used to make a response, and (6) A new memory is formed of the recent event $Q$. 
COMPUTATIONAL MODEL OF INTRUSIVE MEMORIES

At the midnight of a predefined day, indicated as Day 0 , a potentially traumatic event (PTE) is generated and presented to the model. This event is marked as having a greater-thannormal emotional impact $I_{P T E}$, whose precise value of $I_{P T E}$ was manipulated during the simulations.

\section{Memory Representations}

In ACT-R, memories are represented as "chunks", that is, are vector-like structures containing a predefined number of slots, each of which contains one attribute. In general, ACT$\mathrm{R}$ gives programmers much latitude to define memories arbitrarily, using the same format for both episodic and semantic memory representations. The focus of this study, however, is explicitly centered on the retrieval of specific episodic memories. For this reason, we adapted ACT-R representations in the following ways.

In the model, new memories are arbitrary and randomly generated. The structures of these memories are controlled by two parameters, $N$ and $A$, which determine the number of slots in a given memory ( $N$, corresponding to the maximum number of contextual cues $q$ ) and the number of attributes from which a value for a slot can be chosen from $(A$, which determines the richness of the internal representations of events).

Although PTEs might occur in familiar environments and conditions (i.e., partner violence), many traumatic events occur in situations, conditions, and environments that are unique and different from the daily life of the agent; for example, for a war veteran, the environment in which the PTE occurred (a war campaign in a foreign country) might have few points of contact with his or her everyday life back home. The degree of exceptionality of the PTE was captured by parametrically varying the pool of attributes that were chosen for the PTE's slots. Specifically, a congruency parameter $0 \leq C \leq 1$ controlled the proportion of 
COMPUTATIONAL MODEL OF INTRUSIVE MEMORIES

attributes that were unique to the PTE, that is, were selected from a special pool instead of being drawn from the same pool as the attributes of other memories. For $C=0$, the PTE is entirely made of unique attributes, while for $C=1$ the PTE is absolutely indistinguishable from the other randomly generated episodes.

\section{Simulation of Potentially Traumatic Events}

To simulate the effects of emotion and trauma on declarative memory, the ACT-R code was augmented with a set of functions that maintain the scalar value $I(m)$ for every memory $m$ created. Every time a new memory is added, its value $I(m)$ is computed and recorded. The value of $I(m)$ is determined on the basis of the time $t$ at which $i$ is first added to ACT-R's declarative memory system. If $t=I_{P T E}$, then the value of $I(m)$ is set to a predefined value of $I_{P T E}$, which is parametrically manipulated in the following simulations. Otherwise, the value is drawn from a uniform distribution between 0 and 2, so that the mean value of all the non-traumatic memories is $\bar{I}=1$ (and, therefore, $\log (\bar{I})=0$ in Eq. 4).

\section{Contextual Effects}

In ACT-R, the contextual cues $q_{1}, q_{2}, \ldots q_{N}$ are typically held in separate, contentdependent "buffers", each of which represents a different cortical region and provides its own source of spreading activation. Episodic memory, thus, would be best thought of as an aggregate snapshot of the contents of these buffers. For simplicity, the model was simplified so that events and memories are represented in the same format, as a single vector of $N$ attributes. For the sake of simplicity, contextual information and retrieved memories are allocated to different buffers, identified by the red and blue slots in Figure 4. Thus, an event is represented as a context vector $Q$ that is held in the imaginal buffer, and the retrieved memory $m_{T}$ is temporarily placed in the retrieval buffer. After the context has been processed and a response has been made, the current 
$Q$ becomes a new episodic memory that is added to the long-term declarative memory system, and might therefore be retrieved in the future.

Since, in our model, all of the contextual cues are embedded into a single chunk, ACTR's spreading activation function was simplified. Specifically, spreading activation flows from each contextual cue $q_{i}$ to $m$ if the attribute of the $i$-th slot of $m$ is identical to $q$.

\section{Distribution of Contextual and Internally Generated Retrievals}

To create a realistic simulation, the model went through several simulated days of activity in which memory retrieval was either guided by external events (which form the context $Q$ to which a response might be made) or by spontaneous recollection. This latter process is an abstraction for a variety of mental processes that also lead to the retrieval of the PTE, such as rumination, negative appraisal, and even unintentional mind-wandering.

The generation of events that lead to retrievals was modeled as a stochastic process, with the probability of each event being controlled by a gamma distribution over the time of day. Two such distributions were created, one for contextual events and one for the internal unproductive processing events. For a modicum of realism, it was assumed that the contextual events peak in the early morning and are maximally distributed during working hours ( $8 \mathrm{am}-5 \mathrm{pm})$, while unproductive processing events peak at the end of working hours and extend later in the evenings. Figure 6 illustrates the probability densities of the two types of events. Note that, because the cumulative distribution of each curve is 1 , the expected number of events of each type during the day is 1 . To alter the expected number of events during the day, the probability density curves were multiplied by two scaling factors, $E$ (for the events) and $U$ (for unproductive processing). Thus, the scaling factors can be interpreted as the expected number of events of each type occurring within a day. 


\section{Probability of Retrievals During the Day}

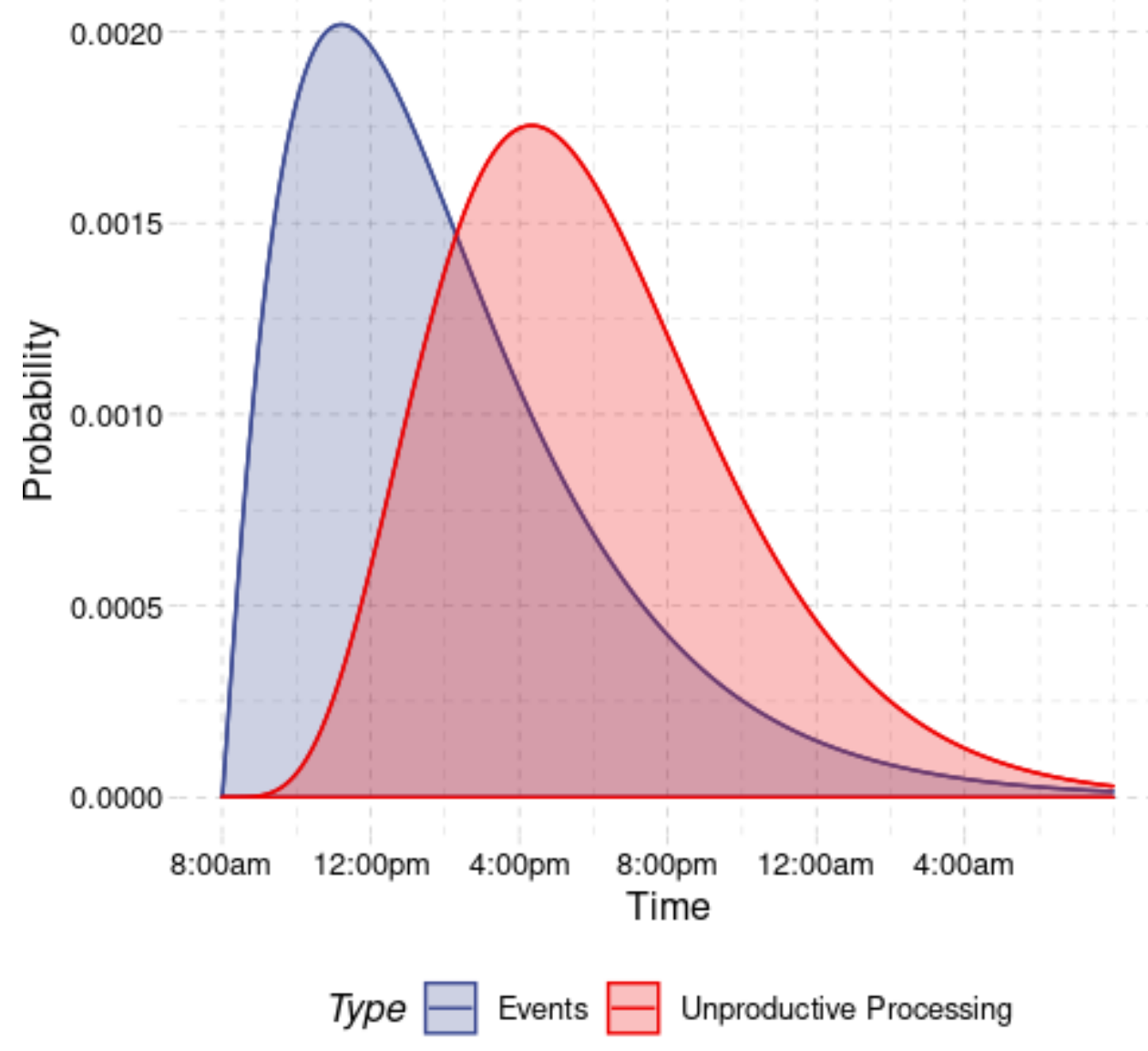

Figure 6: Probability density functions of a retrieval happening because of external events (blue) or because of unproductive processing (red) during each simulated day.

\section{Model Simulations and Data Analysis}

Simulations were run by systematically varying the model parameters as shown in Table 2. The range of these parameters was chosen as follows. The values of $N$ and $A$ were chosen to create a sufficient variety of difference events for the simulations while maintaining the total amount of computations manageable. The values of $W$ were chosen so that its middle value would be equal to $N$ (so that the weight of each cue would be $W / N=1$ in the spreading activation 
equation), and the lower and upper values would correspond to $50 \%$ decrements or increments of this central value. To capture the effect of context, $C$ the range of was divided into four equal intervals, and the first four values that divided. The values of $I$ were evenly space to include a baseline conditions $(I=1)$ and three conditions $(I=20,40$, and 60) in which the net effect of a traumatic event would be comparable to the effect of spreading activation (i.e., $\log (I)=1.3,1.6$, and 1.8, respectively). The values of $U$ were chosen to be either zero, or to match the expected number of retrievals $E$, thus anchoring the range of plausible values. Finally, the values of $\gamma$ were chosen in analogy to the most common values used, in the modeling literature, to capture the temporal discounting factor of future rewards (e.g., Collins, 2018).

For each combination of parameters in parameter space, the model was run for 100 times. Eech run span 160 simulated days, of which 100 occurred before the PTE to populate the model's long-term memory with a minimal history.

The model was implemented using the ACT-R software, version 7.5 (Anderson, 2007). All of the model simulations were run and maintained through GNU Parallel (Tange, 2011). The results of the simulations were analyzed with the R language and statistical software (R Core Team, 2020). Data were visualized using the "ggplot2" package (Wickham, 2011); the model's predictions about structural and functional changes in the brain were visualized using the “ggseg" package (Mowinckel \& Vidal-Piñeiro, 2020). 
Table 2: Summary of the simulation parameters.

\begin{tabular}{|l|l|l|}
\hline Parameter & Meaning & Value or Range \\
\hline$N$ & $\begin{array}{l}\text { Number of features in episodic memories } \\
\text { (cues in event) }\end{array}$ & 8 \\
\hline$A$ & Number of possible attributes for each feature & 4,6 \\
\hline$C$ & Congruency between environment and PTE & $0,0.25,0.5,0.75$ \\
\hline$W$ & $\begin{array}{l}\text { Attentional weight (Working memory } \\
\text { capacity) }\end{array}$ & $0.5,1,1.5$ \\
\hline$d$ & Decay rate & 0.5 (standard decay rate) \\
\hline$I_{P T E}$ & Intensity of PTE & $1,20,40,60$ \\
\hline$E$ & Frequency of event-driven retrievals & 20 \\
\hline$U$ & $\begin{array}{l}\text { Frequency of spontaneous retrievals due to } \\
\text { unproductive processing }\end{array}$ & 0,20 \\
\hline$\gamma$ & Vividness of recalled experience & $0.8,0.9,0.95$ \\
\hline
\end{tabular}

\section{Dependent Variables}

\section{Behavioral Measures}

During each run of the model, the unintential retrieval of intrusive memories during a single day was recorded for up to 60 days after the occurrence of the PTE. For each run, two dependent variables were calculated. The first is the mean probability of retrieving intrusive memories over time, from the day in which the PTE occurred to Day 60. This measure represents an average, ideal trajectory for a specific combination of parameters.

The second measure is distribution of recovery trajectories across all runs within a combination of parameters. To calculate this measure, instead of averaging across all of the recovery curves, the recovery curve of each individual instance of the model is classified in one of the four trajectories discussed in the Introduction (Galatzer-Levy et al., 2018). 
To categorize each run, the following algorithm was used:

1. First, three values are calculated:

a. The mean probability $P_{\text {baseline }}$ of retrieving intrusive memories in the 10 days preceding the PTE, or baseline period. By definition, it is always zero.

b. The mean probability $P_{\text {acute }}$ of retrieving intrusive memories in the 10 days following the PTE, or during the acute period.

c. The mean probability $P_{\text {chronic }}$ of retrieving intrusive memories in the last ten days of the second month after the PTE (i.e, days 51-60 after PTE), or the chronic period.

2. Then, the model uses these three values to calculates two statistical tests:

a. A $t$-test between the baseline and acute period, or acute test.

b. A $t$-test between the acute-period and the chronic period, or chronic test.

3. If the acute test is significant at $p<.05\left(P_{\text {acute }}>P_{\text {baseline }}\right)$, then

a. If the chronic test is also significant at $p<.05$, and $P_{\text {chronic }}>P_{\text {acute }}$, the we classify the trajectory as delayed.

b. If the chronic test is significant at $p<.05$ but $P_{\text {chronic }}<P_{\text {acute }}$, then we classify the trajectory as recovery.

c. If the chronic test is non-significant, then $P_{\text {chronic }} \sim P_{\text {acute }}$, and we classify the trajectory as chronic.

4. If, instead, the acute test is not significant $\left(P_{\text {acute }}=P_{\text {baseline }}\right)$, then:

a. If the chronic test is significant at $p<.05$, then $P_{\text {chronic }}>P_{\text {acute }}$, and we classify the trajectory as delayed. 
COMPUTATIONAL MODEL OF INTRUSIVE MEMORIES

b. If the chronic test is also not significant, then $P_{\text {chronic }}=P_{\text {baseline }}=P_{\text {acute }}$, and we classify the trajectory as resilient.

\section{Neurobiological variables}

A third and final group of dependent variables was collected to see how well the model captures the possible neurobiological effects of traumatic events. Both of these variables were estimated at the chronic period, i.e., Days 51-60 after the PTE.

The first neurobiological dependent variable was the predicted change in hippocampal volume. This was calculated using the entropy equation (Eq. 4) and average the value of $H$ at the end of days 51-60. Specifically, the value of $H$ at the end of each run was compared to the value of $H$ in a baseline condition that would be representative of a healthy, non-traumatized control population. This control condition had parameters that were representative of the general population ( $W=8, C=0.25, \gamma=0.9$; see Results section, below, for how these representative parameters were identified) but was run under no trauma $\left(I_{\mathrm{PTE}}=1\right)$. The percentage difference of each run's entropy $H$ from this baseline condition was taken as the percentage decrease in hippocampus volume.

The second neurobiological dependent variable was the functional connectivity between the medial prefrontal cortex and the hippocampus. This was calculated by measuring the average similarity between the context $Q$ and the retrieved memory during the chronic period, i.e. days 51-60 after the PTE.

\section{Results}

The occurrence of intrusive memories was measured as the probability of retrieving the PTE at any retrieval cycle. For simplicity and ease of interpretation, the results are shown in 
terms of the number of intrusive memory retrievals per simulated day, with the entire simulation $\left(T_{\max }\right)$ extending to approximately two months after the occurrence of the PTE. Figure 7 provides an overview of the results across all parameter space.

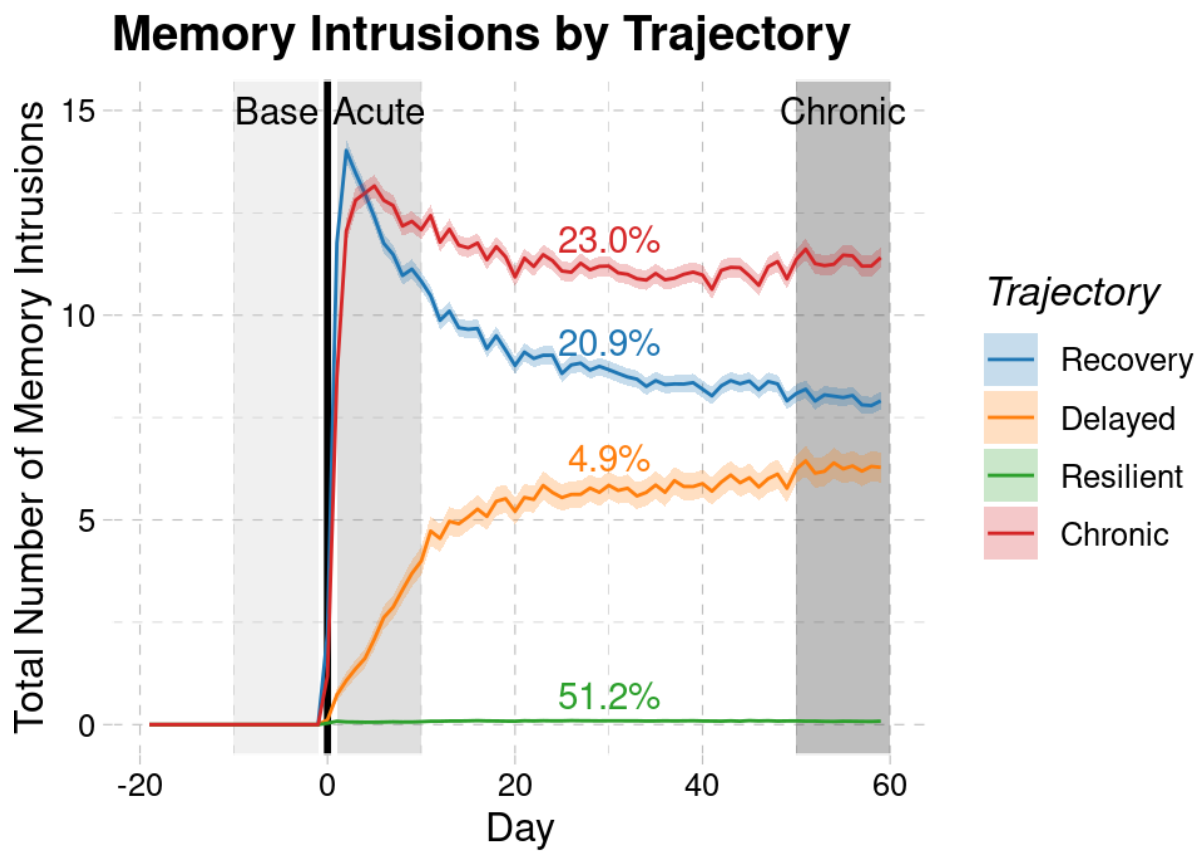

Figure 7. Average timecourse and of traumatic memory intrusions and relative trajectory prevalence for each trajectory type, calculated across all of the model parameter values manipulated in the simulations. The shaded areas represent the time intervals at which the frequency of traumatic memory intrusions is measured to identify each model's run trajectory.

Figure 7 shows the predicted number of memory intrusion across all simulated parameters, divided by the type of recovery trajectories of the corresponding model runs. Neither the proportion of trajectories nor the specific number of traumatic memory intrusions in Figure 7 are representative of the observed effects in the general population. This is because the parameter values were not selected in a way that was empirically informed---in fact, part of the goal of this 
study is to identify empirical parameter values and ranges that better characterize the general population.

To this aim, we examined every combination of parameters to identify the specific set whose proportion of trajectories best represents what is observed in the literature. These proportions were obtained from the meta-analysis reported by Galatzer-Ivy et al. (2018), which gives an estimated prevalence for the Resilient (65.7\%), Recovery (20.8\%), Chronic (10.6\%) and Delayed trajectories (8.9\%) across 54 studies and 76,435 participants. It was found that, for a particular combination of model parameters $\left(C=0.25\right.$, gamma $=0.9, I_{\mathrm{PTE}}=40, W=8$, and $R=$ $0)$, the predicted trajectories closely matched the percentages reported in the meta-analysis. The mean trajectories and they relative percentages are depicted in Figure 8.

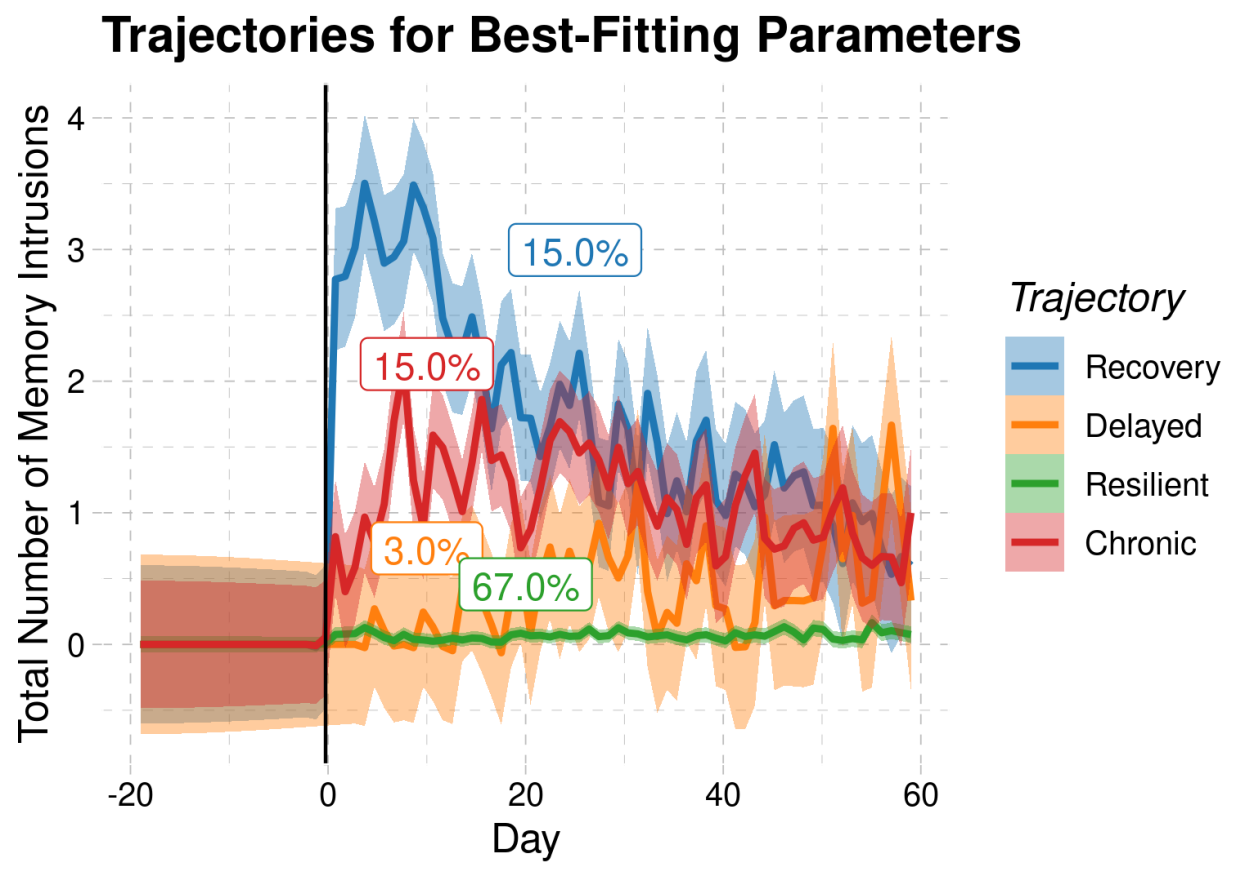

Figure 8. Percentages and timecourses of the different trajectories for the combination of parameters that best matches the prevalence of trajectories in Galatzer-Ivy et al. (2018). 
The percentage of trajectories is not statistically different from what emerged in the metaanalysis $\left(\chi^{2}(3)=7.46, p>.05\right)$ and almost identical to those observed in some recent studies (Whitman et al., 2013).

Having identified a representative set of parameters, we can now examine how systematically varying parameter values that represent different risk factors affect the timecourse and severity of memory intrusions in PTSD, and whether these changes are consistent with the direction of the predicted effects in Table 1 and the empirical findings reported in the literature.

\section{Effects of External Factors}

The external (environmental) factors in our simulations are the intensity $I_{\mathrm{PTE}}$ of the initial traumatic event and the contextual similarity $C$ between the PTE and the current context $Q$ under which a retrieval process is initiated. To examine their effects, an analysis was conducted on all the simulation runs in which the values of idiographic parameters $W$ and gamma and the unproductive processing parameter $R$ were kept at the population-level representative values of Figure 7, while $I$ and $C$ could assume all possible values in Table 1.

Figure 9 illustrates the mean timecourses of traumatic memory intrusions across different values of $I_{\mathrm{PTE}}$ and $C$. 


\section{Effects of PTE Intensity}

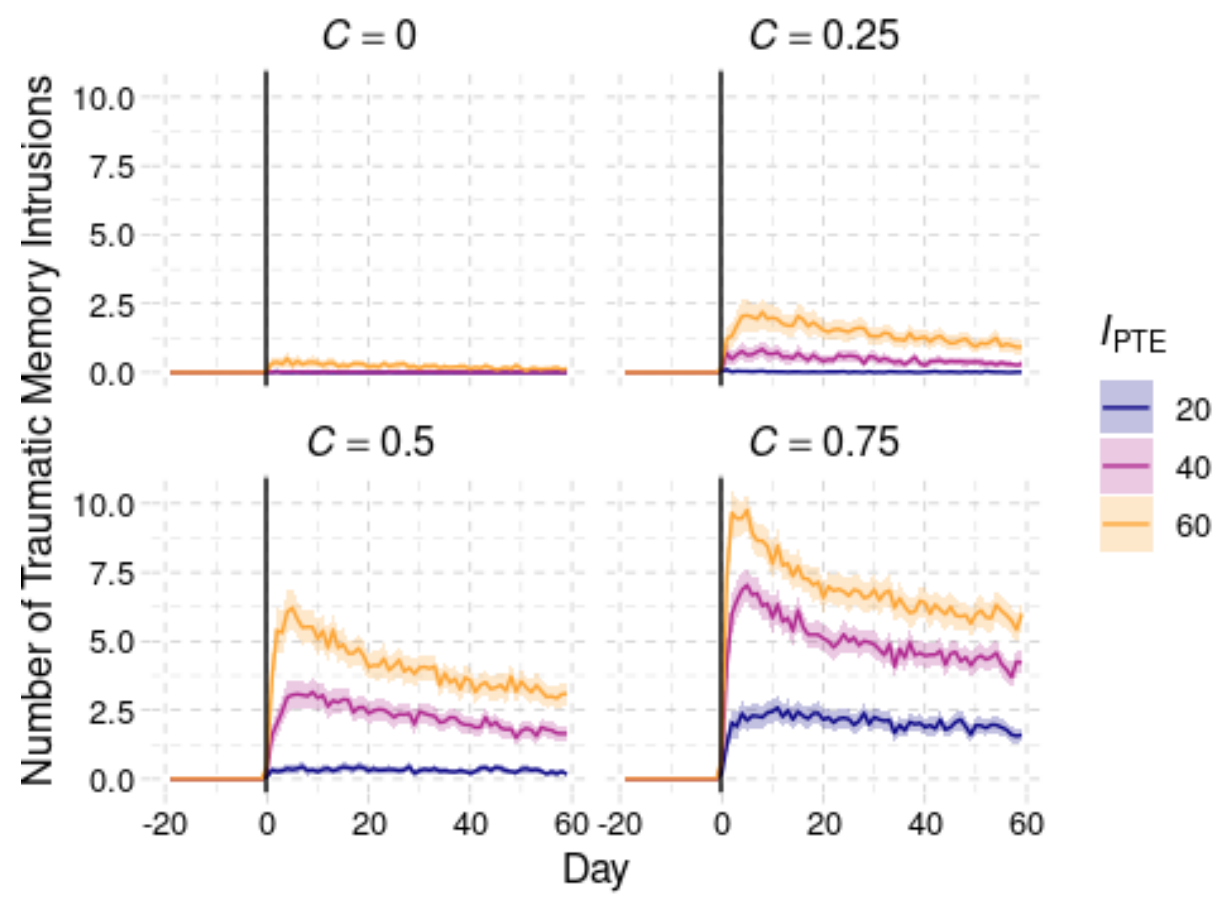

Figure 9: Predicted daily number of intrusions following a traumatic event of different intensity $\left(I_{P T E}\right)$ under varying values of contextual similarity $(C)$.

A number of effects are consistent with the classic results in the literature. In general the frequency of memory intrusions is higher immediately after experiencing the traumatic event, and tends to taper off over time; this fact is consistent with the prevalence of spontaneous recovery across studies (Galatzer-Levy et al., 2018). The frequency of memory intrusions is systematically larger after events of greater traumatic intensity. Additionally, and unsurprisingly, the number of intrusions tends to be higher when the environment contains a greater number of multiple cues associated with the traumatic event, as modeled by the contextual similarity parameter $C$.

To systematically investigate the effects of $I$ and $C$, the average number of memory intrusions $N$ at the end of the simulated period of time (Days 51-60) was modeled through a 
COMPUTATIONAL MODEL OF INTRUSIVE MEMORIES

generalized fixed-effect linear model of the form $N \sim \beta_{0}+\beta_{\mathrm{I}} I_{P T E}+\beta_{\mathrm{C}} C+\beta_{\mathrm{I}, \mathrm{C}} I_{P T E} C+\varepsilon$. Because the number of intrusions $N$ is a count variable, the statistical model used a Poisson rather than Gaussian link function. The statistical analysis shows a significant effect of both the PTE intensity (standardized $\beta_{\mathrm{I}}=0.005, p<0.001$ ) and context (standardized $\beta_{\mathrm{C}}=0.008, p<0.001$ ). Their interaction was also significant with a negative parameter value $\left(\beta_{\mathrm{I}, \mathrm{C}}=-0.003, \mathrm{p}<0.001\right)$.

Although they provide important quantitative information, each of the timecourses of Figure 7 is averaged from hundreds of individual runs of the model, each of which would exhibit its own recovery trajectory. We predicted that both the intensity of the traumatic event and the proportion of traumatic cues in the environment would have significant effects on the model's recovery trajectories, with the number of resilient trajectories declining as the values of $I$ and $C$ increase. The relative distribution of the other three types of trajectories (Recovery, Chronic, and Delayed), however, is harder to predict because of the possible interactions between factors and the fact that each trajectory's prevalence is constrained by the relative frequency of the others. Indeed, this is precisely the type of situation in which computer simulations might provide insight in the balance of forces that affect recovery.

Figure 10 depicts the prevalence of each of the four trajectories for each combination of $I_{\mathrm{PTE}}$ and $C$. The relative distribution of the four trajectories was analyzed using a MANOVA model. Note that, because the prevalence of the trajectories is bound to sum up to $100 \%$, this statistical model has only three effective dependent variables, since the fourth trajectory's prevalence is completely determined once the first three are known. For the purpose of our analysis, the three dependent variables were the prevalences of the Resilient, Recovery, and Delayed trajectories; the statistical results would not change if any other set of three trajectories were considered. 


\section{Effect of External Factors on Trajectories}

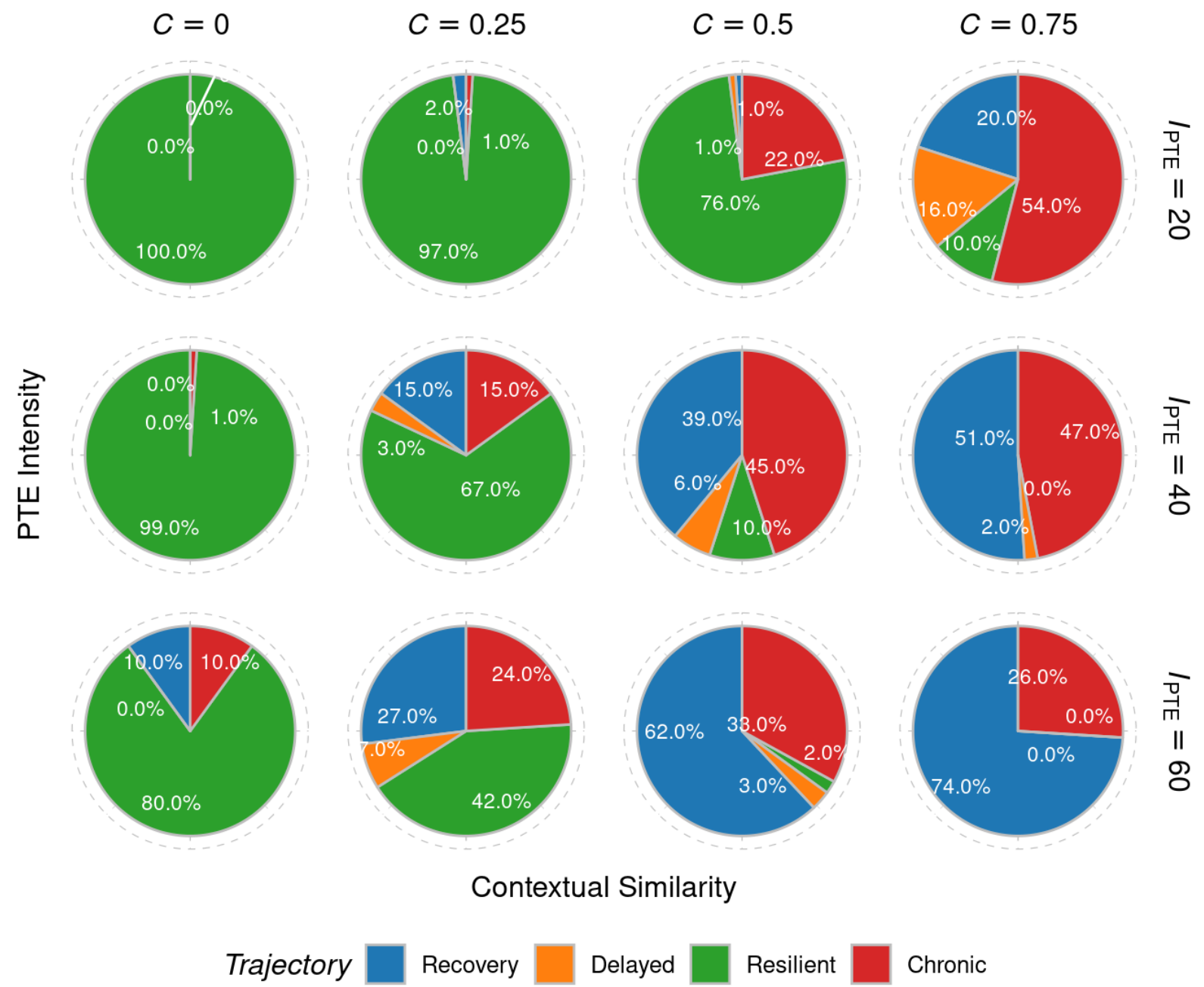

Figure 10: Relative proportion of the four fundamental recovery trajectories (Figure under different values of the intensity of the traumatic event (PTE) and the contextual similarity between the traumatic event and daily life.

The MANOVA analysis revealed that that both the intensity of the PTE (Pillai's trace = $0.25, F(3,1196)=133.05, p<0.0001)$ and its contextual similarity $C$ (Pillai's trace $=0.54, F(3$, $1196)=460.64, p<0.0001)$ as well as their interaction (Pillai's trace $=0.06, F(3,1196)=24.93$, $p<0.0001)$ significantly affected the distribution of the trajectories. 
Because an increase in the prevalence of one trajectory must occur at the expense of the others, as can be seen in Figure 9, the differential share of different trajectories responds differently to different factors. For instance, the Resilient trajectory steadily declines as the values of $I_{\mathrm{PTE}}$ and $C$ increase, while the Delayed trajectory waxes and wanes.

To further analyze this effect, four separate analyses were conducted, each of which estimated the probability that a run of the model under different conditions exhibited a specific trajectory. All of the analyses were implemented as a generalized linear model of the form $T=\beta_{0}$ $+\beta_{\mathrm{I}} I_{P T E}+\beta_{\mathrm{C}} C+\beta_{\mathrm{I}, \mathrm{C}} I_{P T E} C+\varepsilon$, with $T$ being a binary variable (coded as $T=1$ if the specific run exhibits the target trajectory and $T=0$ otherwise) and the model using a binomial link function.

In the case of the Resilient trajectory (green in Figure 9), both the intensity of the PTE (standardized $\left.\beta_{\mathrm{I}}=-3.41, p<0.00001\right)$ and its contextual similarity $\left(\beta_{\mathrm{C}}=-5.78, p<0.00001\right)$ had significant and negative effects. Furthermore, their interaction did not reach significance $\left(\beta_{\mathrm{I}, \mathrm{C}}=\right.$ $0.39, p>0.59$ ), implying that their effects were additive. The other three trajectories, however, show a more complicated pattern. The main effects of both factors were significant and positive for all three trajectories (standardized $\beta_{\mathrm{I}}>2.94, p<0.001 ; \beta_{\mathrm{C}}>3.28, p<0.0001$ ); this reflects the deeper and longer-lasting effects of the PTE, which increase the probability of Recovery, Delayed, and Chronic trajectories while decreasing the share of Resilient ones. The interaction term, however, was significant only for the Delayed and Chronic trajectories. In both cases, the interaction has a negative sign $\left(\beta_{\mathrm{I}, \mathrm{C}}<-4.27, p<0.0001\right)$ impling that, as the intensity of the PTE and its contextual similarity increases, the probability of these trajectories might actually decrease (as it does in Figure 9). It is also worth noting that, when the environmental context shares very few attributes with the original traumatic event (i.e., $C=0.0$ or 0.25 ), resilient trajectories remain common even for high levels of traumatic intensity. This is compatible with 
the potentially paradoxical finding that resilient trajectories are more common in combattraumatized veterans, despite the severity of the events witnessed (Galatzer-Levy et al., 2018).

In summary, the model's simulations largely reflect the patterns observed in the traumatic stress literature, showing consistent effects of both the intensity of the trauma and the presence of traumatic cues in the environment as powerful drivers of intrusive memories and pathological trajectories.

\section{Effects of Idiographic Factors}

Next, we will examine the effects of idiographic characteristics, which in our model are abstracted in the cognitive control parameter $W$ and the intensity of relived experience $\gamma$. As shown in Figure 10, both factors significantly affected the onset and timecourse of intrusive memories

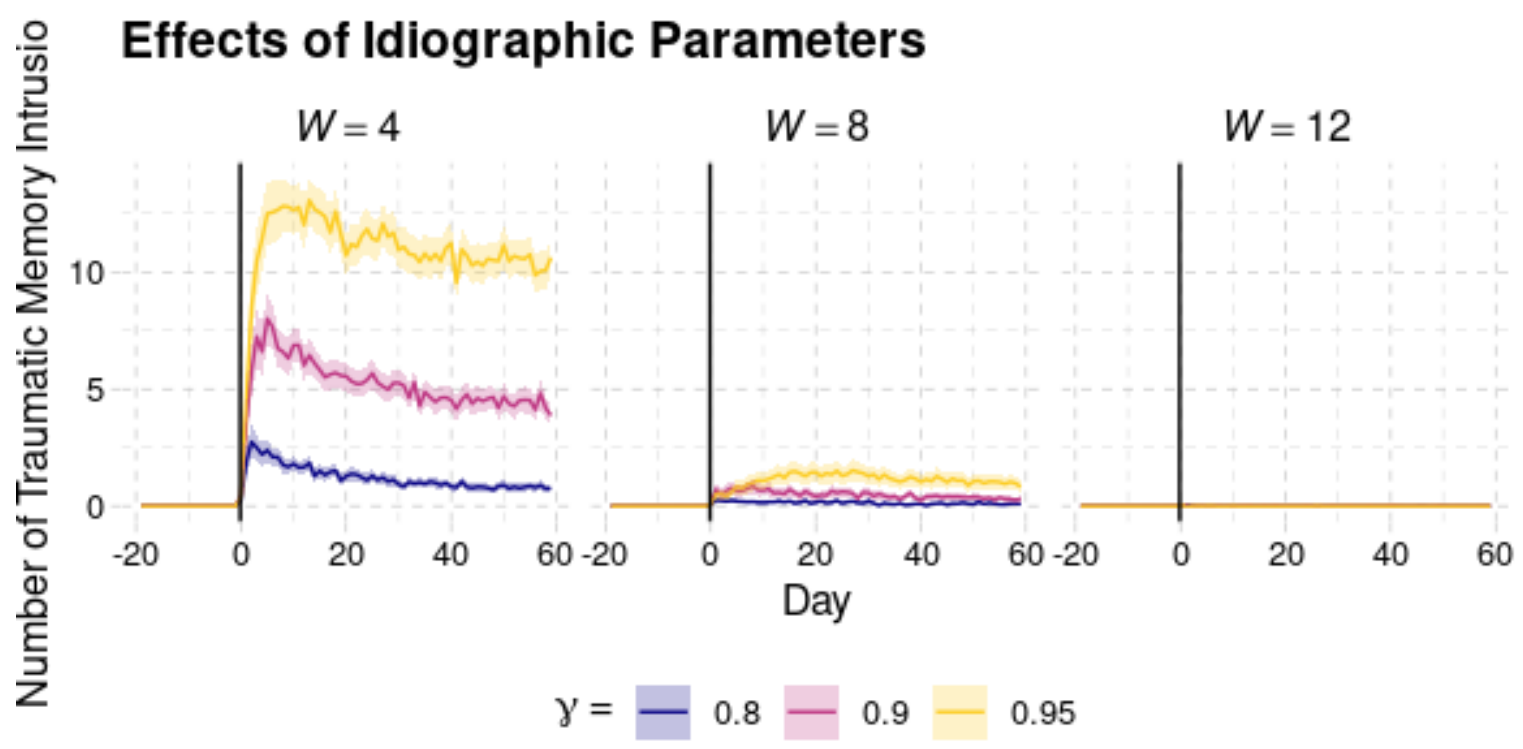

Figure 11: Effects of idiographic factors of cognitive control (working memory capacity) and intrusive memory intensity (gamma) on daily memory intrusions. 
COMPUTATIONAL MODEL OF INTRUSIVE MEMORIES

In general, the effects of the cognitive control factor $W$ were much stronger than expected, with the model registering no intrusive memories when $W=12$. This suggests that the range chosen for $W$ was unrealistically large, a fact that will need to be taken into consideration in future simulation studies.

As in the case of external factors, the effects of the $W$ and $\gamma$ on the number of traumatic events was analyzed by through a generalized linear model of the form $N=\beta_{0}+\beta_{\mathrm{W}} W+\beta_{\gamma} \gamma+$ $\beta_{\mathrm{W}, \gamma} W \gamma+\varepsilon$ with a Poisson link function, with $N$ being the cumulative sum of intrusive memories in Days 51-60 after the PTE. As expected, the analysis showed that increased levels of cognitive control were associated with less frequent intrusive memories $\left(\beta_{\mathrm{W}}=-0.005, p=0.01\right)$, while increased levels of vividness were associated with more frequent intrusions $\left(\beta_{\gamma}=0.002, p\right.$ $<0.0001)$. The two factors did not interact with each other $\left(\beta_{\mathrm{W}, \gamma}=-0.0002, p>0.91\right)$.

As before, the prevalence of trajectories was analyzed with a MANOVA model, using the proportion of Resilient, Recovery, and Delayed trajectories as the dependent variables (the proportion of Chronic trajectories was determined once the other three are known) and the values of $W$ and $\gamma$ as the independent variables. Both $W$ (Pillai's trace $=0.62, F(3,896)=476.34, p<$ $0.0001)$ and $\gamma($ Pillai's trace $=0.08, F(3,896)=26.56, p<0.0001)$, as well as their interaction (Pillai's trace $=0.05, F(3,896)=14.97, p<0.0001)$, were statistically significant, with $W$ explaining the most variance, as indicated by its large Pillai's trace value.

Four separate analyses were conducted on the probability of each model run resulting in one of the four trajectories; all of them used a generalized linear model with a logistic link function. Because of the large variance in the proportion of trajectories (due to the massive effects of $W$ : Figure 11), none of the interaction terms were significant. Thus, we analyzed all four trajectories with a simpler generalized linear model of the form $N=\beta_{0}+\beta_{\mathrm{W}} W+\beta_{\gamma} \gamma+\varepsilon$. 
Increases in the cognitive control parameter $W$ were found to be significantly associated with increases in the Resilient trajectory (standardized $\beta_{\mathrm{W}}=6.86, p<0.00001$ ) and with decreases in the probability of Recovery, Delayed, and Chronic trajectories (standardized $\beta_{\mathrm{W}}<-3.63, p<$ 0.00001). This is consistent with the hypothesis that executive control increases resilience to traumatic events, likely because of the increased ability to control emotional memories (Kaldewaij et al., 2021). Increases in the recollection vividness $\gamma$, on the other hand, were found to be significantly associated with increases in the occurrences of Chronic and Delayed trajectories (standardized $\beta_{\gamma}>1.43, p<0.00001$ ) and decreases in the occurrences of Recovery and Resilient trajectories (standardized $\beta_{\gamma}<-0.90, p<0.001$ ). This is consistent with our hypothesis that recollection vividness negatively affects recovery by perpetuating the lingering effects of traumatic memories.

In summary, both lower cognitive control $(W)$ and higher intensity of intrusive memories $(\gamma)$ were associated with more intrusive memories and generally more pathological outcome trajectories. The large effect of $W$ is likely due to the fact that the range of parameter values examined was too large. However, while the distribution of trajectories for very high values of $W$ seem unrealistic, the distribution of trajectories and the severity of memory intrusions for very low values of $W$ is compatible with those found in cases of intellectual disability (Mevissen $\&$ de Jongh, 2010). 


\section{Effect of Idiographic Factors on Trajectories}

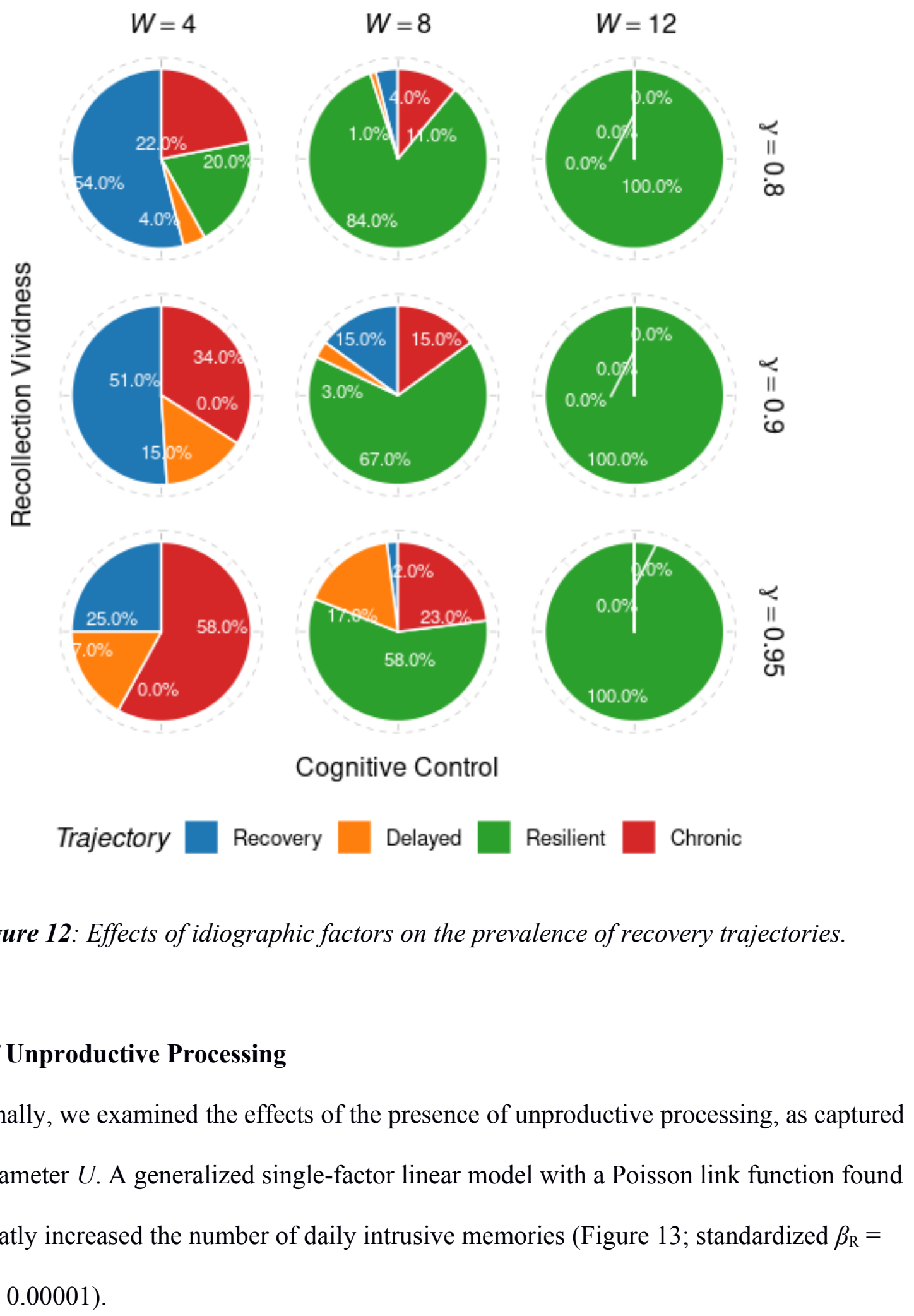

\section{Effects of Unproductive Processing}

Finally, we examined the effects of the presence of unproductive processing, as captured by the parameter $U$. A generalized single-factor linear model with a Poisson link function found that $U$ greatly increased the number of daily intrusive memories (Figure 13; standardized $\beta_{\mathrm{R}}=$ $0.003, p<0.00001)$ 


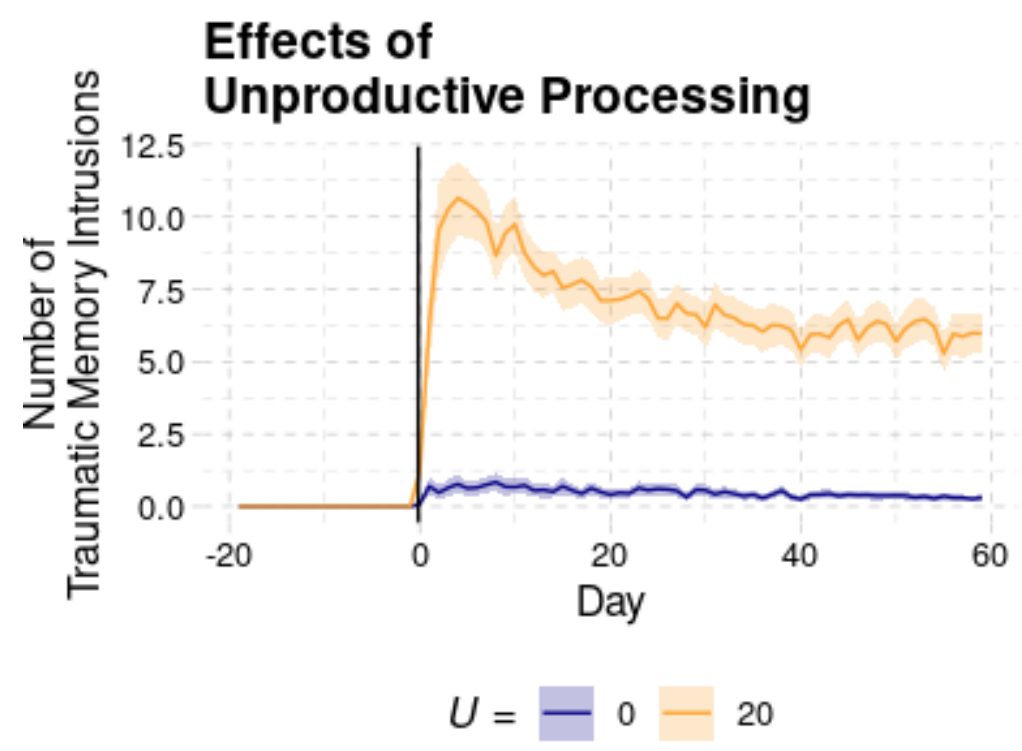

Figure 13: Effects of unproductive processing on the frequency of daily memory intrusions

A MANOVA found that it also significantly affected the distribution of trajectories (Figure 14: Pillai's trace $=0.45 ; F(1,196)=55.09, p<0.0001)$. Separate analysis of for each trajectory, using a generalized linear model with a logistic link function, showed that unproductive processing negatively affected the probability of Resilient trajectory (standardized $\beta_{\mathrm{R}}=-4.38, p<$ 0.00001), significantly increased the probability of occurrence of Chronic and Recovery trajectories (standardized $\beta_{\mathrm{R}}>1.41, p<0.0005$ ), but had no effect on the Delayed trajectory (standardized $\beta_{\mathrm{R}}=0.81, p>0.70$ ). 


\section{Effect of Unproductive Processing on Trajectories}
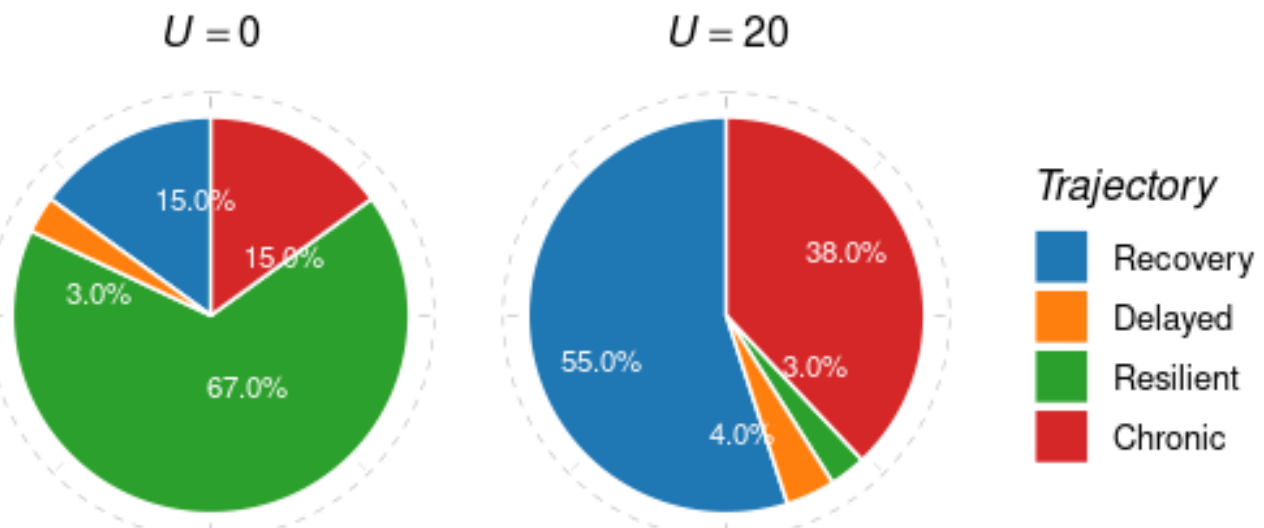

Unproductive Processing

Figure 14: Effects of unproductive processing on the proportion of recovery trajectories.

\section{Neurobiological Effects of PTSD}

In addition to the behavioral effects, the model also generated predictions about how intrusive memories affect the brain. Specifically, we will focus on PTSD-related changes in the size of the hippocampus and in its functional connectivity to the prefrontal cortex. These two measures capture consistent and key abnormalities reported in the literature, and they can be approximated in the model as the value of Shannon's information entropy, $H$, across all memories (Smith et al., 2021) and the mean value of the similarity between the retrieved memory and the contextual cues.

Although the clinical literature typically reports the prevalence of PTSD symptoms and recovery trajectories after a particular type of traumatic event, neuroimaging studies of PTSD largely focus on differences between groups of individuals who already exhibit different symptoms. They could be, for example, individuals suffering from PTSD vs. healthy matched controls with no history of trauma or, more commonly, individuals exhibiting PTSD symptoms 
vs individuals not exhibiting PTSD symptoms after a similar, traumatic event. Because in these studies the two groups were matched with respect to some of the factors of interest (such as the intensity of the traumatic event or individual differences in executive function, corresponding to our parameters $I$ and $W$ ), it would be uninformative to conduct an analysis of the effects of these factors. It is also problematic that the traumatic events the two groups were exposed to are considered "similar": as noted in the beginning, the intensity of a traumatic event interacts with the characteristics of the individual, and group assignments often fail to account for other important confounding factors, such as other trauma history. Furthermore, in the majority of studies, no information is given as to the specific trajectory of the individuals in the experimental group: while they were currently exhibiting PTSD symptoms (that is why they were assigned to the group), their symptoms could be on a recovery course, or chronically stable, or they could have had a delayed onset.

To conduct a proper analysis that is more comparable with the literature, we analyzed the model's neurobiological dependent variables using each run's end-point trajectory, that is, whether each model run's intrusive memories on days 51-60 after the PTE is significantly greater than zero or not in a $t$-test. This lumps models runs that either never developed intrusive memories (Resilient) or successfully recovered after developing them (Recovery) into the "Control" group, and runs that either experienced intrusive memories throughout the run (Chronic) or developed them later on (Delayed) in the "PTSD" group. Note that this analysis departs from our previously reported work (Smith et al., 2021) which instead focused on the main effect of one external (intensity of the traumatic event, $I_{\mathrm{PTE}}$ ) and one internal (cognitive control, $W$ ) factor.

Figure 15 illustrates the results of this analysis for hippocampal volume. The simulated 
PTSD group's change in hippocampus volume $(M=-8.63 \%, S D=7.82 \%)$ was significantly larger than the traumatized control group's $(M=-0.04 \%, S D=1.47 \%$; Welch two-sample test $t(12754)=92.42, p<0.0001)$, with the control group's change in hippocampus being not significantly different than zero (one-sample $t(3097)=-1.50, p>0.13$ ).

Hippocampus Volume Decrease by Experimental Group
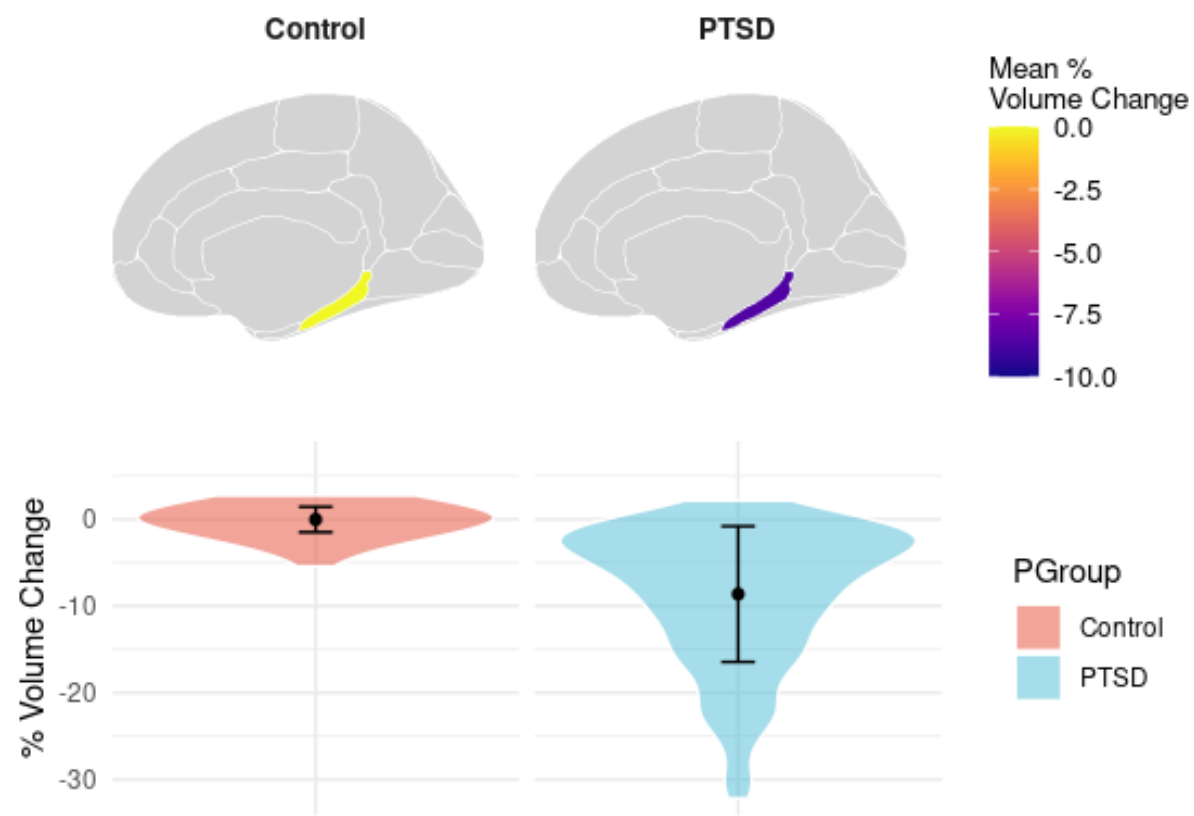

Figure 15. Predicted changes in hippocampal volume by experimental group. In the violin density plots (bottom), points represent means and error bars represent standard deviations.

A similar analysis was conducted for the predicted functional connectivity between the medial prefrontal cortex and the hippocampus, operationalized as the degree of similarity between retrieved memories and current context and visualized in Figure 16. Because, in our simulations, the similarity between the traumatic memory and the context is experimentally 
manipulated as the factor $C$, this analysis was restricted to the subset of simulations in which $C=$ 0 . The results showed that the simulated traumatized control group's runs (in which the model that were exposed to trauma but successfully recovered) exhibited significantly greater functional connectivity $(M=0.53, S D=0.20)$ than the simulated PTSD group $(M=0.12, S D=$ 0.15; Welch two-sample test, $t(722.86)=39.84, p<0.0001)$.

Functional Connectivity Between Medial PFC and Hippocampus by Experimental Group

Control
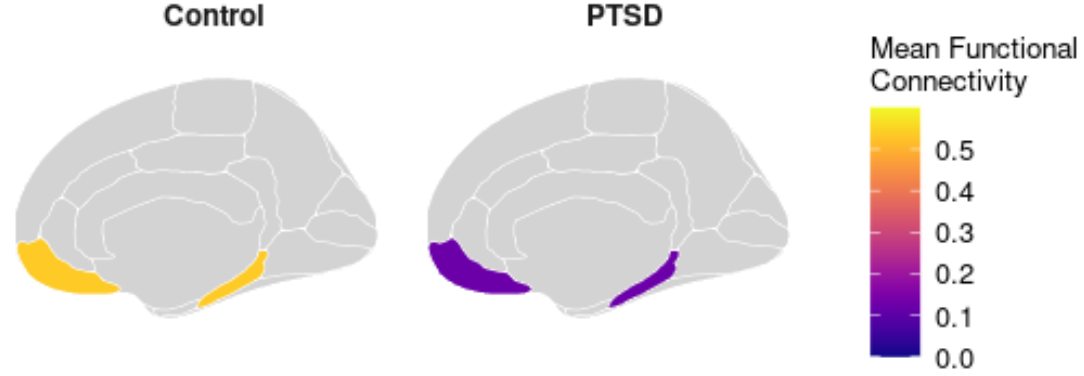

PTSD

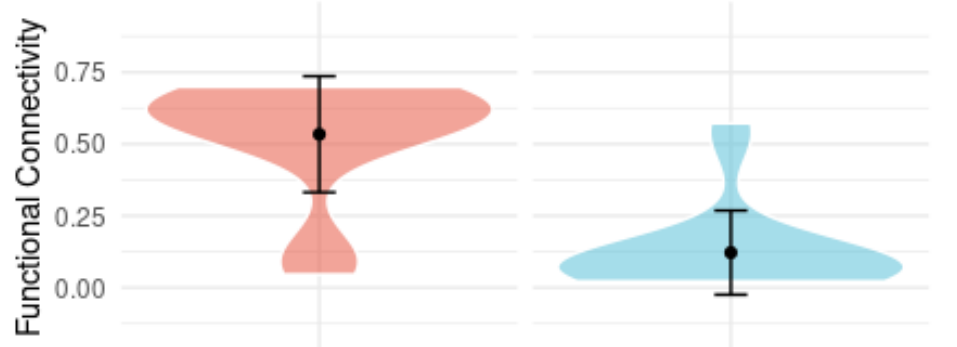

PGroup

Control

PTSD

Figure 16. Predicted changes in functional connectivity between ventromedial PFC and hippocampus, divided by the simulated group of patients. In the violin density plots (bottom), points represent means and error bars represent standard deviations. 


\section{Discussion}

In this paper, we have described a computational model of intrusive memories following a traumatic event. The model is rooted in the rational analysis framework of human memory (Anderson, 1990) and accounts for the insurgence of traumatic memories as a consequence of the additional activation boost that their emotional intensity confers. The model correctly predicts a number of behavioral findings, including the frequency of intrusive memories after the traumatic event and the effects that external and idiographic factors have on individual recovery trajectories. Additionally, the model also predicts a reduction in the size of the hippocampus and a reduction in the functional connectivity between prefrontal cortex and the hippocampus as a consequence of the persistence of intrusive memories. To the best of our knowledge, this is the first model to provide such a large coverage of neuroimaging and clinical findings.

The model predictions are noteworthy not only in extent but also in detail. The use of a realistic memory model and extended simulation times allow for detailed day prediction of the occurrence of intrusive memories under different contexts. In doing so, the work significantly tests the reach of the ACT-R declarative memory model to a timescales many orders of magnitudes larger than that of the simple laboratory tasks it was designed for. The fact the model could run and produce meaningful and realistic results on this extended timescale, even when the parameters were left unchanged (as it was the case of the decay rate $d$ ), testifies to the robustness of this model. Such modeling over days, weeks, months, and years will be needed to extend this work to clinical applications.

\section{Limitations}

One of the main limitations of the model is the narrow way in which it captures learning. In our model, learning only occurs as a function of the general laws that govern memory decay 
over time, such as the power law of forgetting and the effects of recency and frequency. In contrast, previous models have focused on a fear avoidance and learned cued associations (Radell et al., 2017), both of which were captured and expressed in terms of reinforcement and associative learning, respectively. Although our results show that the laws of memory decay and their modulation by emotion go a long way to capture a variety of behavioral and neurological phenomena, the effects of associative learning should not be discounted and certainly play an important role. For example, clinical psychotherapeutic interventions for PTSD are both successful and largely based on the associative tenets of the fear learning and extinction (Cusack et al., 2016; Graham \& Milad, 2011; Lebois et al., 2019). Thus, these two approaches to modeling PTSD should not be taken as mutually exclusive, but as two different venues that focus on different facets of the disorder.

A related limitation is that our model addresses only intrusive memories per se, but does not address the emotional and arousal states associated with intrusive memories, nor does it capture the behavioral changes that are associated with them. Intrusions themselves are not pathological and can differ on both frequency and distress. While frequency and distress are likely highly associated, it is possible to have intrusions without distress, with the later not being pathological. Further, patients suffering from PTSD often report persistent anxiety and distress and modify their routines to actively avoid triggers. These changes have been previously addressed by the associative type of computational model (Radell et al., 2015) but are outside the reach of the model presented herein.

A word of caution should be also spent in comparing the model's behavioral predictions against the empirical data. This is because the samples involved in different studies are highly heterogeneous, varying significantly in the type of traumatic event and the time between the 
event and subsequent measurements. Our model captures the heterogeneity of the events only within a bidimensional space (intensity $I$ and similarity to everyday context $C$ ), but it is likely that other dimensions might be needed to explain, for example, the differential effects of interpersonal trauma exposure and participation of sectarian violence (Kessler et al., 2017). Similarly, our model only captures the dynamics of recovery within a limited timeframe of two months, but different studies have reported data collected much beyond that horizon, and up to $20+$ years after the traumatic event.

Another limitation of our model is that parameters are not allowed to vary as a function of trauma exposure or memory content. For example, the model currently assumes that a individual cognitive control or working memory capacity (captured by the parameter $W$ ) remains stable after the PTE, while experimental evidence suggests that it might be compromised by exposure to trauma (El-Hage et al., 2006).

Perhaps most remarkably, the parameter $d$, which controls the degree of memory decay and forgetting, was kept constant not only within but also across simulations. This choice was motivated by the fact that little is still known about individual differences in forgetting (although work quantifying such differences has recently been done: Sense et al., 2018; 2016; Zhou et al., 2021) and, as a consequence, not much is known about the interaction between forgetting and intrusive memories. Nonetheless, because of its critical role in determining the frequency of intrusive memories, future work should include careful examinations of this parameter's role.

The most common explanation for the reduction of hippocampal volume is that prolonged stress leads to hippocampal damage, for example through the accumulation of stressrelated hormones like cortisol. However, as noted by Garfinkel and Liberzon (2009), cortisol levels are not universally or consistently elevated in individuals with PTSD; indeed, potentially 
even suppressed (Morris et al., 2016; Pan et al., 2018). The model's estimates, instead, rely on a different logic, namely, that the hippocampus size reflects the landscape of memory availability and that, in PTSD, the dominant availability of the traumatic memory implicitly causes a reduced number of hippocampal cells and synapses devoted to storing memory information (Smith et al., 2021).

Finally, one last limitation that needs to be acknowledged is the duration of the simulations after the PTE. Because of their computational demands, our simulations only extended to 60 days after trauma. Clinical consensus suggests that trajectories would stabilize at a later time period ( $>90$ days). Because of this, our algorithm for trajectory classification takes into account the ascending or descending trend in the number of traumatic memory intrusions, rather than testing for a complete recovery at the end-point. This fact might explain the paradoxical results of Figure 10, where recovery trajectories seem to increase with the intensity $I_{\mathrm{PTE}}$ of the traumatic event. Thus, an extended simulation horizon in which the number of intrusive memories after three or more months would likely yield better and more reliable estimates of the trajectories.

\section{Conclusions}

These limitations notwithstanding, we believe that this computational model represents a first and important step into better understanding the nature of traumatic memories and their relationship to posttraumatic stress disorder and memory processing. Models such as this have the ability to manipulate parameters identified in the clinical literature and link them to behavioral and neurobiological effects, showing and confirming functional relationships that simply are not possible to manipulate in the real world. Critically, they have the ability to capture and model dynamic retrieval processes characterized by episodic event processing. 
Indeed, strong predictors emerged. Both higher event severity and congruence with the current environment resulted in higher intrusions. Similarly, lower working memory capacity and higher post-event unproductive processing such as rumination also resulted in higher intrusions. This convergence with the clinical literature and their emergence from functional, computational modeling of memory argues beyond simply Granger causality in the clinical literature that these factors likely have causal properties.

Finally, this model might have relevant impacts in mental health provision. Despite remarkable advances, the field of computational psychiatry (Huys et al., 2016; Montague et al., 2012; Wiecki et al., 2015) has fallen short of the possibility of delivering individually parametrized models that could be realistically used to aid in treating patients. However, the degree of detail and realism achieved by these simulations, in both their timescale and the detail of behavioral and neural predictions, make it possible to consider using this model in realistic mental health applications. For example, this model could be calibrated on individual data and provide mobile health recommendation, data collection, and monitoring (Pirolli et al., 2017; Springer et al., 2018), which could be key in the case of disorders, like PTSD, where identifying in the immediate aftermath of a potentially traumatic event those who are most likely to have chronic psychopathology and should receive triaged intervention has been elusive and barriers to receiving early intervention high, even despite having impairing symptoms (Shalev et al., 2011). We see this as an exciting venue for future research.

\section{Acknowledgments}

This research was supported by a scholarship from the University of Washington's Institute for Neuroengineering (UWIN) to BMS a from a Royalty Research Fund award (grant \# A142764) from the University of Washington to EP and LZ. 


\section{References}

Akiki, T. J., Averill, C. L., \& Abdallah, C. G. (2017). A Network-Based Neurobiological Model of PTSD: Evidence From structural and functional Neuroimaging Studies. Current Psychiatry Reports, 19(11), 81.

Alvarez, P., \& Squire, L. R. (1994). Memory consolidation and the medial temporal lobe: a simple network model. Proceedings of the National Academy of Sciences of the United States of America, 91(15), 7041-7045.

Anderson, J. R. (1974). Retrieval of propositional information from long-term memory. Cognitive Psychology, 6(4), 451-474.

Anderson, J. R. (1983). A spreading activation theory of memory. Journal of Verbal Learning and Verbal Behavior, 22(3), 261-295.

Anderson, J. R. (1990). The Adaptive Character of Thought. Mahwah, NJ: Lawrence Erlbaum Associates.

Anderson, J. R. (2007). How Can the Mind Occur in the Physical Universe? New York, NY: Oxford University Press.

Anderson, J. R., Bothell, D., Byrne, M. D., Douglass, S., Lebiere, C., \& Qin, Y. (2004). An integrated theory of the mind. Psychological Review, 111(4), 1036-1060.

Anderson, J. R., Bothell, D., Lebiere, C., \& Matessa, M. (1998/5). An Integrated Theory of List Memory. Journal of Memory and Language, 38(4), 341-380.

Anderson, J. R., Fincham, J. M., Qin, Y., \& Stocco, A. (2008). A central circuit of the mind. 
Trends in Cognitive Sciences, 12(4), 136-143.

Anderson, J. R., \& Schooler, L. J. (1991). Reflections of the environment in memory. Psychological Science, 2(6), 396-408.

Baddeley, A. (2010). Working memory. Current Biology: CB, 20(4), R136-R140.

Baddeley, A. D., \& Logie, R. H. (1999). Working memory: The multiple-component model. In A. Miyake \& P. Shah (Eds.), Models of working memory: Mechanisms of active maintenance and executive control (pp. 28-61). Cambridge University Press.

Badre, D., \& Wagner, A. D. (2007). Left ventrolateral prefrontal cortex and the cognitive control of memory. Neuropsychologia, 45(13), 2883-2901.

Berntsen, D., Willert, M., \& Rubin, D. C. (2003). Splintered memories or vivid landmarks? Qualities and organization of traumatic memories with and without PTSD. Applied Cognitive Psychology, 17(6), 675-693.

Besnard, A., \& Sahay, A. (2016). Adult Hippocampal Neurogenesis, Fear Generalization, and Stress. Neuropsychopharmacology: Official Publication of the American College of Neuropsychopharmacology, 41(1), 24-44.

Bomyea, J., \& J. Lang, A. (2015). The Role of Executive Functioning in PTSD and its Treatment. Current Psychiatry Reviews, 11(3), 160-171.

Bremner, J. D., Randall, P., Scott, T. M., Bronen, R. A., Seibyl, J. P., Southwick, S. M., Delaney, R. C., McCarthy, G., Charney, D. S., \& Innis, R. B. (1995). MRI-based measurement of hippocampal volume in patients with combat-related posttraumatic stress disorder. The 
COMPUTATIONAL MODEL OF INTRUSIVE MEMORIES

American Journal of Psychiatry, 152(7), 973-981.

Bremner, J. D., Randall, P., Vermetten, E., Staib, L., Bronen, R. A., Mazure, C., Capelli, S., McCarthy, G., Innis, R. B., \& Charney, D. S. (1997). Magnetic resonance imaging-based measurement of hippocampal volume in posttraumatic stress disorder related to childhood physical and sexual abuse--a preliminary report. Biological Psychiatry, 41(1), 23-32.

Burgess, G. C., Gray, J. R., Conway, A. R. A., \& Braver, T. S. (2011). Neural mechanisms of interference control underlie the relationship between fluid intelligence and working memory span. Journal of Experimental Psychology. General, 140(4), 674-692.

Bywaters, M., Andrade, J., \& Turpin, G. (2004). Intrusive and non-intrusive memories in a nonclinical sample: The effects of mood and affect on imagery vividness. Memory, 12(4), 467478.

Cahill, L., Gorski, L., \& Le, K. (2003). Enhanced human memory consolidation with postlearning stress: interaction with the degree of arousal at encoding. Learning \& Memory , $10(4), 270-274$.

Cohodes, E. M., Chen, S. H., Lieberman, A. F., \& Bush, N. R. (2020). Examination of the associations between young children's trauma exposure, trauma-symptomatology, and executive function. Child Abuse \& Neglect, 108, 104635.

Collins, A. G. E. (2018). The Tortoise and the Hare: Interactions between Reinforcement Learning and Working Memory. Journal of Cognitive Neuroscience, 30(10), 1422-1432.

Collins, A. M., \& Loftus, E. F. (1975). A spreading-activation theory of semantic processing. Psychological Review, 82(6), 407. 
Cusack, K., Jonas, D. E., Forneris, C. A., Wines, C., Sonis, J., Middleton, J. C., Feltner, C., Brownley, K. A., Olmsted, K. R., Greenblatt, A., Weil, A., \& Gaynes, B. N. (2016). Psychological treatments for adults with posttraumatic stress disorder: A systematic review and meta-analysis. Clinical Psychology Review, 43, 128-141.

Daily, L. Z., Lovett, M. C., \& Reder, L. M. (2001). Modeling individual differences in working memory performance: a source activation account. Cognitive Science, 25(3), 315-353.

Dancy, C. L. (2013). ACT-RФ: A cognitive architecture with physiology and affect. Biologically Inspired Cognitive Architectures, 6, 40-45.

Danker, J. F., \& Anderson, J. R. (2010). The ghosts of brain states past: remembering reactivates the brain regions engaged during encoding. Psychological Bulletin, 136(1), 87-102.

Danker, J. F., Gunn, P., \& Anderson, J. R. (2008). A rational account of memory predicts left prefrontal activation during controlled retrieval. Cerebral Cortex, 18(11), 2674-2685.

El-Hage, W., Gaillard, P., Isingrini, M., \& Belzung, C. (2006). Trauma-related deficits in working memory. Cognitive Neuropsychiatry, 11(1), 33-46.

Frans, O., Rimmö, P.-A., Aberg, L., \& Fredrikson, M. (2005). Trauma exposure and posttraumatic stress disorder in the general population. Acta Psychiatrica Scandinavica, 111(4), 291-299.

Fum, D., \& Stocco, A. (2004). Memory, Emotion, and Rationality: An ACT-R interpretation for Gambling Task results. In C. D. Schunn, M. C. Lovett, C. Lebiere \& P. Munro (Ed.), Proceedings of the 6th International Conference on Cognitive Modeling (pp. 106-111). Lawrence Erlbaum Associates. 
Galatzer-Levy, I. R., Huang, S. H., \& Bonanno, G. A. (2018). Trajectories of resilience and dysfunction following potential trauma: A review and statistical evaluation. Clinical Psychology Review, 63, 41-55.

Garfinkel Sarah N., \& Liberzon Israel. (2009). Neurobiology of PTSD: A review of neuroimaging findings. Psychiatric Annals, 39(6).

Gilbertson, M. W., Shenton, M. E., Ciszewski, A., Kasai, K., Lasko, N. B., Orr, S. P., \& Pitman, R. K. (2002). Smaller hippocampal volume predicts pathologic vulnerability to psychological trauma. Nature Neuroscience, 5(11), 1242-1247.

Graham, B. M., \& Milad, M. R. (2011). The study of fear extinction: implications for anxiety disorders. The American Journal of Psychiatry, 168(12), 1255-1265.

Hackmann, A., Ehlers, A., Speckens, A., \& Clark, D. M. (2004). Characteristics and content of intrusive memories in PTSD and their changes with treatment. Journal of Traumatic Stress, $17(3), 231-240$.

Hagenaars, M. A., van Minnen, A., Hoogduin, C. A. L., \& Verbraak, M. (2009). A transdiagnostic comparison of trauma and panic memories in PTSD, panic disorder, and healthy controls. Journal of Behavior Therapy and Experimental Psychiatry, 40(3), 412422.

Hardt, O., Nader, K., \& Nadel, L. (2013). Decay happens: the role of active forgetting in memory. Trends in Cognitive Sciences, 17(3), 111-120.

Huys, Q. J. M., Maia, T. V., \& Frank, M. J. (2016). Computational psychiatry as a bridge from neuroscience to clinical applications. Nature Neuroscience, 19(3), 404-413. 
Juvina, I., Larue, O., \& Hough, A. (2018). Modeling valuation and core affect in a cognitive architecture: The impact of valence and arousal on memory and decision-making. Cognitive Systems Research, 48, 4-24.

Kaldewaij, R., Koch, S. B. J., Hashemi, M. M., Zhang, W., Klumpers, F., \& Roelofs, K. (2021). Anterior prefrontal brain activity during emotion control predicts resilience to post-traumatic stress symptoms. Nature Human Behaviour, 1-10.

Kane, M. J., \& Engle, R. W. (2002). The role of prefrontal cortex in working-memory capacity, executive attention, and general fluid intelligence: an individual-differences perspective. Psychonomic Bulletin \& Review, 9(4), 637-671.

Kane, M. J., \& Engle, R. W. (2003). Working-memory capacity and the control of attention: the contributions of goal neglect, response competition, and task set to Stroop interference. Journal of Experimental Psychology. General, 132(1), 47-70.

Kessler, R. C., Aguilar-Gaxiola, S., Alonso, J., Benjet, C., Bromet, E. J., Cardoso, G., Degenhardt, L., de Girolamo, G., Dinolova, R. V., Ferry, F., Florescu, S., Gureje, O., Haro, J. M., Huang, Y., Karam, E. G., Kawakami, N., Lee, S., Lepine, J.-P., Levinson, D., ... Koenen, K. C. (2017). Trauma and PTSD in the WHO World Mental Health Surveys. European Journal of Psychotraumatology, 8(sup5), 1353383.

Koenigs, M., \& Grafman, J. (2009). Posttraumatic stress disorder: the role of medial prefrontal cortex and amygdala. The Neuroscientist: A Review Journal Bringing Neurobiology, Neurology and Psychiatry, 15(5), 540-548.

Kolassa, I.-T., Ertl, V., Eckart, C., Kolassa, S., Onyut, L. P., \& Elbert, T. (2010). Spontaneous 
COMPUTATIONAL MODEL OF INTRUSIVE MEMORIES

remission from PTSD depends on the number of traumatic event types experienced. Psychological Trauma: Theory, Research, Practice and Policy, 2(3), 169-174.

Kotseruba, I., \& Tsotsos, J. K. (2020). 40 years of cognitive architectures: core cognitive abilities and practical applications. Artificial Intelligence Review, 53(1), 17-94.

Lebois, L. A. M., Seligowski, A. V., Wolff, J. D., Hill, S. B., \& Ressler, K. J. (2019).

Augmentation of extinction and inhibitory learning in anxiety and trauma-related disorders. Annual Review of Clinical Psychology, 15, 257-284.

Ledoux, J. (1998). The Emotional Brain: The Mysterious Underpinnings of Emotional Life. Simon and Schuster.

LeDoux, J. (2012). Rethinking the emotional brain. Neuron, 73(4), 653-676.

Logue, M. W., van Rooij, S. J. H., Dennis, E. L., Davis, S. L., Hayes, J. P., Stevens, J. S., Densmore, M., Haswell, C. C., Ipser, J., Koch, S. B. J., \& Others. (2018). Smaller hippocampal volume in posttraumatic stress disorder: A multisite ENIGMA-PGC study: subcortical volumetry results from posttraumatic stress disorder consortia. Biological Psychiatry, 83(3), 244-253.

Lovett, M. C., Daily, L. Z., \& Reder, L. M. (2000). A source activation theory of working memory: cross-task prediction of performance in ACT-R. Cognitive Systems Research, 1(2), 99-118.

Marks, E. H., Franklin, A. R., \& Zoellner, L. A. (2018). Can’t get it out of my mind: A systematic review of predictors of intrusive memories of distressing events. Psychological bulletin, 144(6), 584. 
McClelland, J. L., McNaughton, B. L., \& O’Reilly, R. C. (1995). Why there are complementary learning systems in the hippocampus and neocortex: Insights from the successes and failures of connectionist models of learning and memory. Psychological Review, 102(3), 419.

McGaugh, J. L. (2000). Memory--a century of consolidation. Science, 287(5451), 248-251.

Mevissen, L., \& de Jongh, A. (2010). PTSD and its treatment in people with intellectual disabilities: a review of the literature. Clinical Psychology Review, 30(3), 308-316.

Michael, T., Ehlers, A., Halligan, S. L., \& Clark, D. M. (2005). Unwanted memories of assault: what intrusion characteristics are associated with PTSD? Behaviour Research and Therapy, $43(5), 613-628$.

Miyake, A., Friedman, N. P., Emerson, M. J., Witzki, A. H., Howerter, A., \& Wager, T. D. (2000). The unity and diversity of executive functions and their contributions to complex “Frontal Lobe" tasks: A latent variable analysis. Cognitive Psychology, 41(1), 49-100.

Montague, P. R., Dolan, R. J., Friston, K. J., \& Dayan, P. (2012). Computational psychiatry. Trends in Cognitive Sciences, 16(1), 72-80.

Morina, N., Leibold, E., \& Ehring, T. (2013). Vividness of general mental imagery is associated with the occurrence of intrusive memories. Journal of Behavior Therapy and Experimental Psychiatry, 44(2), 221-226.

Morris, M. C., Hellman, N., Abelson, J. L., \& Rao, U. (2016). Cortisol, heart rate, and blood pressure as early markers of PTSD risk: A systematic review and meta-analysis. Clinical Psychology Review, 49, 79-91. 
Moscovitch, M., Rosenbaum, R. S., Gilboa, A., Addis, D. R., Westmacott, R., Grady, C., McAndrews, M. P., Levine, B., Black, S., Winocur, G., \& Nadel, L. (2005). Functional neuroanatomy of remote episodic, semantic and spatial memory: a unified account based on multiple trace theory. Journal of Anatomy, 207(1), 35-66.

Mowinckel, A. M., \& Vidal-Piñeiro, D. (2020). Visualization of Brain Statistics With R Packages ggseg and ggseg3d. Advances in Methods and Practices in Psychological Science, $3(4), 466-483$

Murty, V. P., FeldmanHall, O., Hunter, L. E., Phelps, E. A., \& Davachi, L. (2016). Episodic memories predict adaptive value-based decision-making. Journal of Experimental Psychology. General, 145(5), 548-558.

Murty, V. P., Tompary, A., Adcock, R. A., \& Davachi, L. (2017). Selectivity in Postencoding Connectivity with High-Level Visual Cortex Is Associated with Reward-Motivated Memory. The Journal of Neuroscience: The Official Journal of the Society for Neuroscience, 37(3), 537-545.

Myers, C. E., Moustafa, A. A., Sheynin, J., Vanmeenen, K. M., Gilbertson, M. W., Orr, S. P., Beck, K. D., Pang, K. C. H., \& Servatius, R. J. (2013). Learning to obtain reward, but not avoid punishment, is affected by presence of PTSD symptoms in male veterans: empirical data and computational model. PLOS One, 8(8), e72508.

Nadel, L., \& Moscovitch, M. (1997). Memory consolidation, retrograde amnesia and the hippocampal complex. Current Opinion in Neurobiology, 7(2), 217-227.

Nadel, L., Samsonovich, A., Ryan, L., \& Moscovitch, M. (2000). Multiple trace theory of human 
COMPUTATIONAL MODEL OF INTRUSIVE MEMORIES

memory: computational, neuroimaging, and neuropsychological results. Hippocampus, $10(4), 352-368$.

Nelson, M. D., \& Tumpap, A. M. (2017). Posttraumatic stress disorder symptom severity is associated with left hippocampal volume reduction: a meta-analytic study. CNS Spectrums, 22(4), 363-372.

O’Doherty, D. C. M., Chitty, K. M., Saddiqui, S., Bennett, M. R., \& Lagopoulos, J. (2015). A systematic review and meta-analysis of magnetic resonance imaging measurement of structural volumes in posttraumatic stress disorder. Psychiatry Research, 232(1), 1-33.

Ohman, A., \& Mineka, S. (2001). Fears, phobias, and preparedness: Toward an evolved module of fear and fear learning. Psychological Review, 108(3), 483-522.

Panksepp, J. (2004). Affective Neuroscience: The Foundations of Human and Animal Emotions. Oxford University Press.

Pan, X., Wang, Z., Wu, X., Wen, S. W., \& Liu, A. (2018). Salivary cortisol in post-traumatic stress disorder: A systematic review and meta-analysis. BMC Psychiatry, 18(1), 324.

Patil, A., Murty, V. P., Dunsmoor, J. E., Phelps, E. A., \& Davachi, L. (2017). Reward retroactively enhances memory consolidation for related items. Learning \& Memory, 24(1), $65-69$.

Pavlik, P. I., \& Anderson, J. R. (2008). Using a model to compute the optimal schedule of practice. Journal of Experimental Psychology. Applied, 14(2), 101-117.

Pavlik, P. I., Jr, \& Anderson, J. R. (2005). Practice and forgetting effects on vocabulary memory: 
An activation-based model of the spacing effect. Cognitive Science, 29(4), 559-586.

Peverill, M., Finn, A. S., \& McLaughlin, K. A. (2017). Dimensions of childhood adversity have distinct associations with neural systems underlying executive functioning. Development and Psychopathology, 29(5), 1777-1794.

Pirolli, P., Mohan, S., Venkatakrishnan, A., Nelson, L., Silva, M., \& Springer, A. (2017). Implementation intention and reminder effects on behavior change in a mobile health system: A predictive cognitive model. Journal of Medical Internet Research, 19(11), e397.

Pitman, R. K., Gilbertson, M. W., Gurvits, T. V., May, F. S., Lasko, N. B., Metzger, L. J., Shenton, M. E., Yehuda, R., Orr, S. P., \& Harvard/VA PTSD Twin Study Investigators. (2006). Clarifying the origin of biological abnormalities in PTSD through the study of identical twins discordant for combat exposure. Annals of the New York Academy of Sciences, 1071, 242-254.

Posner, J., Russell, J. A., \& Peterson, B. S. (2005). The circumplex model of affect: an integrative approach to affective neuroscience, cognitive development, and psychopathology. Development and Psychopathology, 17(3), 715-734.

Radell, M. L., Beck, K. D., Pang, K. C. H., \& Myers, C. E. (2015). Using signals associated with safety in avoidance learning: computational model of sex differences. PeerJ, 3, e1081.

Radell, M. L., Myers, C. E., Sheynin, J., \& Moustafa, A. A. (2017). Computational models of posttraumatic stress disorder (PTSD). Computational Models of Brain and Behavior, 43, 4355.

Radell, M. L., Sanchez, R., Weinflash, N., \& Myers, C. E. (2016). The personality trait of 
COMPUTATIONAL MODEL OF INTRUSIVE MEMORIES

behavioral inhibition modulates perceptions of moral character and performance during the trust game: behavioral results and computational modeling. PeerJ, 4, e1631.

Ranganath, C., \& Ritchey, M. (2012). Two cortical systems for memory-guided behaviour. Nature Reviews. Neuroscience, 13(10), 713-726.

R Core Team. (2020). R: A Language and Environment for Statistical Computing. R Foundation for Statistical Computing. https://www.R-project.org/

Redish, A. D. (2004). Addiction as a computational process gone awry. Science, 306(5703), 1944-1947.

Rice, P. J., \& Stocco, A. (2021). Estimating individual differences in working memory through ACT-R modeling and resting state connectivity. Proceedings of the 19th International Conference on Cognitive Modeling.

Roediger, H. L. III, Balota, D. A., \& Watson, J. M. (2001). Spreading activation and arousal of false memories. In H. L. Roediger III, J. S. Nairne, I. Neath, \& A. M. Surprenant (Eds.), The nature of remembering: Essays in honor of Robert G. Crowder (pp. 95-115). American Psychological Association. https://doi.org/10.1037/10394-006

Roelofs, A. (1992). A spreading-activation theory of lemma retrieval in speaking. Cognition, 42(1-3), 107-142.

Roxanne Sopp, M., Streb, M., Brueckner, A. H., Schäfer, S. K., Lass-Hennemann, J., Mecklinger, A., \& Michael, T. (2021). Prospective associations between intelligence, working memory capacity, and intrusive memories of a traumatic film: Potential mediating effects of rumination and memory disorganization. Journal of Behavior Therapy and 
Experimental Psychiatry, 70, 101611.

Rubin, D. C., Berntsen, D., \& Bohni, M. K. (2008). A memory-based model of posttraumatic stress disorder: evaluating basic assumptions underlying the PTSD diagnosis. Psychological Review, 115(4), 985-1011.

Saunders, N., Downham, R., Turman, B., Kropotov, J., Clark, R., Yumash, R., \& Szatmary, A. (2015). Working memory training with tDCS improves behavioral and neurophysiological symptoms in pilot group with post-traumatic stress disorder (PTSD) and with poor working memory. Neurocase, 21(3), 271-278.

Sense, F., Behrens, F., Meijer, R. R., \& van Rijn, H. (2016). An individual's rate of forgetting is stable over time but differs across materials. Topics in Cognitive Science, 8(1), 305-321.

Sense, F., Meijer, R. R., \& van Rijn, H. (2018). Exploration of the rate of forgetting as a domainspecific individual differences measure. Frontiers in Education, 3, 112.

Shalev, A. Y., Ankri, Y. L. E., Peleg, T., Israeli-Shalev, Y., \& Freedman, S. (2011). Barriers to receiving early care for PTSD: results from the Jerusalem trauma outreach and prevention study. Psychiatric Services , 62(7), 765-773.

Shannon, C. E. (1948). A mathematical theory of communication. The Bell System Technical Journal, 27(3), 379-423.

Sheynin, J., Moustafa, A. A., Beck, K. D., Servatius, R. J., \& Myers, C. E. (2015). Testing the role of reward and punishment sensitivity in avoidance behavior: a computational modeling approach. Behavioural Brain Research, 283, 121-138. 
Shin, L. M., Orr, S. P., Carson, M. A., Rauch, S. L., Macklin, M. L., Lasko, N. B., Peters, P. M., Metzger, L. J., Dougherty, D. D., Cannistraro, P. A., Alpert, N. M., Fischman, A. J., \& Pitman, R. K. (2004). Regional cerebral blood flow in the amygdala and medial prefrontal cortex during traumatic imagery in male and female Vietnam veterans with PTSD. Archives of General Psychiatry, 61(2), 168-176.

Shin, L. M., Rauch, S. L., \& Pitman, R. K. (2006). Amygdala, medial prefrontal cortex, and hippocampal function in PTSD. Annals of the New York Academy of Sciences, 1071, 67-79.

Shin, L. M., Wright, C. I., Cannistraro, P. A., Wedig, M. M., McMullin, K., Martis, B., Macklin, M. L., Lasko, N. B., Cavanagh, S. R., Krangel, T. S., Orr, S. P., Pitman, R. K., Whalen, P. J., \& Rauch, S. L. (2005). A functional magnetic resonance imaging study of amygdala and medial prefrontal cortex responses to overtly presented fearful faces in posttraumatic stress disorder. Archives of General Psychiatry, 62(3), 273-281.

Smith, B. M., Thomasson, M., Yang, Y. C., Sibert, C., \& Stocco, A. (2021). When fear shrinks the brain: A computational model of the effects of posttraumatic stress on hippocampal volume. Topics in Cognitive Science, 13(3), 499-514. https://doi.org/10.1111/tops.12537

Springer, A., Venkatakrishnan, A., Mohan, S., Nelson, L., Silva, M., \& Pirolli, P. (2018). Leveraging self-affirmation to improve behavior change: a mobile health app experiment. JMIR mHealth and uHealth, 6(7), e157.

Stocco, A., \& Anderson, J. R. (2008). Endogenous control and task representation: an fMRI study in algebraic problem-solving. Journal of Cognitive Neuroscience, 20(7), 1300-1314.

Sutton, R. S., \& Barto, A. G. (1998). Reinforcement learning: An introduction (Vol. 1). 
COMPUTATIONAL MODEL OF INTRUSIVE MEMORIES

Cambridge, MA: MIT Press,.

Tange, O. (2011). GNU Parallel-the command-line power tool. The USENIX Magazine, 36(1), $42-47$.

Thompson-Schill, S. L., D’Esposito, M., Aguirre, G. K., \& Farah, M. J. (1997). Role of left inferior prefrontal cortex in retrieval of semantic knowledge: A reevaluation. Proceedings of the National Academy of Sciences of the United States of America, 94(26), 14792-14797.

van Rooij, S. J. H., \& Jovanovic, T. (2019). Impaired inhibition as an intermediate phenotype for PTSD risk and treatment response. Progress in Neuro-Psychopharmacology \& Biological Psychiatry, 89, 435-445.

van Rooij, S. J. H., Rademaker, A. R., Kennis, M., Vink, M., Kahn, R. S., \& Geuze, E. (2014). Impaired right inferior frontal gyrus response to contextual cues in male veterans with PTSD during response inhibition. Journal of Psychiatry \& Neuroscience: JPN, 39(5), 330-338.

Villarreal, G., Hamilton, D. A., Petropoulos, H., Driscoll, I., Rowland, L. M., Griego, J. A., Kodituwakku, P. W., Hart, B. L., Escalona, R., \& Brooks, W. M. (2002). Reduced hippocampal volume and total white matter volume in posttraumatic stress disorder. Biological Psychiatry, 52(2), 119-125.

Weber, D. L., Clark, C. R., McFarlane, A. C., Moores, K. A., Morris, P., \& Egan, G. F. (2005). Abnormal frontal and parietal activity during working memory updating in post-traumatic stress disorder. Psychiatry Research, 140(1), 27-44.

White, C. N., Congdon, E., Mumford, J. A., Karlsgodt, K. H., Sabb, F. W., Freimer, N. B., London, E. D., Cannon, T. D., Bilder, R. M., \& Poldrack, R. A. (2014). Decomposing 
decision components in the stop-signal task: a model-based approach to individual differences in inhibitory control. Journal of Cognitive Neuroscience, 26(8), 1601-1614.

White, C. N., Curl, R. A., \& Sloane, J. F. (2016). Using decision models to enhance investigations of individual differences in Cognitive Neuroscience. Frontiers in Psychology, $7,81$.

Whitman, J. B., North, C. S., Downs, D. L., \& Spitznagel, E. L. (2013). A prospective study of the onset of PTSD symptoms in the first month after trauma exposure. Annals of Clinical Psychiatry: Official Journal of the American Academy of Clinical Psychiatrists, 25(3), 163172.

Wickham, H. (2011). ggplot2. Wiley Interdisciplinary Reviews. Computational Statistics, 3(2), $180-185$.

Wiecki, T. V., Poland, J., \& Frank, M. J. (2015). Model-Based Cognitive Neuroscience Approaches to Computational Psychiatry: Clustering and Classification. Clinical Psychological Science, 3(3), 378-399.

Zhou, P., Sense, F., van Rijn, H., \& Stocco, A. (2021). Reflections of idiographic long-term memory characteristics in resting-state neuroimaging data. Cognition, 212, 104660. 